\title{
Accurate, grid-robust and versatile combined- field discretization for the electromagnetic scattering analysis of perfectly conducting targets
}

\author{
Eduard Ubeda, Ivan Sekulic, Juan M. Rius and Alex Heldring
}

\begin{abstract}
Recent implementations of the Electric-Field Integral Equation (EFIE) for the electromagnetic scattering analysis of perfectly conducting targets rely on the electric current expansion with the monopolar-RWG basis functions, discontinuous across mesh edges, and the field testing over volumetric subdomains attached to the surface boundary triangulation. As compared to the standard RWG-based EFIE-approaches, normally continuous across edges, these schemes exhibit enhanced versatility, allowing the analysis of geometrically non-conformal meshes, and improved accuracy, especially for subwavelength sharp-edged conductors. In this paper, we present a monopolar-RWG discretization by the Method of Moments (MoM) of the Combined-Field Integral Equation (CFIE) resulting from the addition of a volumetrically tested discretization of the EFIE and the Galerkin tested MFIE-implementation. We show for sharp-edged conductors the degree of improved accuracy in the computed RCS and the convergence properties in the iterative search of the solution. More importantly, as we show in the paper, these implementations become in practice advantageous because of their robustness to flaws in the grid generation or their agility in handling complex meshes arising from the interconnection of independently meshed domains. The hybrid RWG/monopolar-RWG discretization of the CFIE defines the RWG discretization over geometrically conformal and smoothly varying mesh regions and inserts the monopolar-RWG expansion strictly at sharp edges, for improved accuracy purposes, or over boundary lines between partitioning mesh domains, for the sake of enhanced versatility. These hybrid schemes offer similar accuracy as their fully monopolar-RWG counterparts but with fewer unknowns and allow naturally non-conformal mesh transitions without inserting additional inter-domain continuity conditions or new artificial currents.
\end{abstract}

Index Terms - Combined-Field Integral Equation, radar cross section, Method of Moments, Basis functions, computational electromagnetics, interior resonance problem.

\section{Introduction}

The numerical analysis of the electromagnetic wave scattering from perfectly conducting (PEC) objects with the Electric-Field Integral Equation (EFIE) or the Magnetic-Field Integral Equation (MFIE) suffers from the so-called interior resonance problem at some particular frequencies [1]. These are eigenfrequencies of the homogeneous equations where the cavity problems yield source-free solutions (resonant cavity modes). The solution of the outer scattering problem then becomes non-unique because of the emergence of a nontrivial solution to the interior problem [2]. In consequence, the condition numbers of the resulting impedance matrices rise and the accuracy of the computed solutions deteriorate. The Combined-Field Integral Equation (CFIE), arising from the linear combination of the EFIE and the MFIE, excels as an integral equation free from internal resonances [3]. For this reason, the CFIE is normally preferred over the EFIE or the MFIE for the full-wave scattering analysis of conductors modelled through closed surfaces, especially for bodies with big enough electrical dimensions, which allow the definition of cavity resonant fields [2].

The conventional discretization of the EFIE by the method of moments (MoM) gives rise to Galerkin schemes with divergence-conforming basis functions, such as the low-order RWG set, which ensure the normal continuity of the current across edges [4]. This is advantageous in computational terms because the hypersingular contributions of the kernel cancel out and the resulting kernel becomes weakly singular. In addition, these edge-based schemes are conforming (i.e., with converging solutions in the physical space of currents). However, the application of such basis functions is restricted to geometrically conformal meshes, with all pairs of adjacent facets sharing a single edge. As is well known, the edge-based schemes require primarily, prior to the numerical analysis, the search for all the 
interior edges [5] arising from the discretization (shared between pairs of adjacent triangles). These routines therefore ignore the remaining boundary edges (linked exclusively to one triangle each) [6]. Nowadays the widespread use of full-wave Integral-Equation (IE) codes has set these edge-search procedures as indispensable. However, these routines become inappropriate for some types of meshes; namely, defective, with slits between facets or with unconnected vertices, or non-conformal, with pairs of adjacent facets not meeting in a single edge. The adoption of the conventional edge-search procedures [6] for defective meshes, originating from flaws in the mesh generation, results in an incomplete identification of the set of interior edges (some of them wrongly tagged as boundary edges), which translates into a wrong modeling of the currents. On the other hand, non-conformal meshes are increasingly required nowadays for the modular analysis and design of complex objects, such as aircrafts, ships, or multiple-component devices, conveniently decomposed into independently meshed regions [7]. The generation of a conformal mesh for high-definition structures, composed of parts with different electrical sizes or electromagnetic properties, may represent a complicated engineering task. Additionally, from the programmer's point of view, the generation of the impedance matrix in the RWG-analysis of composite objects requires remarkable bookkeeping effort in order to provide for the proper continuity conditions around junction edges, where boundary interfaces of several regions intersect [8].

The monopolar-RWG basis functions establish a discontinuous transition across edges in the expansion of the current [9]. These are facet-based schemes because they rely strictly on facets, thereby ignoring edges. As compared to the conventional edge-based schemes, they excel as versatile tools, amenable to a wide variety of meshes, gridrobust [10] and agile in the management of junctions [11], [12]. The facet-based implementations, just like other point-based schemes [13],[14], give rise to nonconforming expansions because the expanded currents, with no interfacet continuity constraints, do not belong to the physical space of currents. The monopolar-RWG schemes provide improved flexibility with respect to the RWG-schemes at the expense of increasing the number of unknowns. The successful adoption of RWG-schemes on geometrically conformal meshes for decades was justified in a context of restricted computational resources, such that the definition of additional unknowns was just too costly. Over the past years, though, the monopolar-RWG schemes have gained attention from researchers thanks to the significant increase on available memory resources.

A monopolar-RWG discretization of the EFIE with Galerkin testing would require the evaluation of the hypersingular contributions from the double gradient of the Green's function through elaborate routines [15]. Alternatively, the integration by parts of the double gradient kernel contributions leads to contour-surface or doublesurface integrals, easy-to-manage, and to double-contour integrals, unbounded for self-or edge-adjacent interactions. Recently, several strategies have been put forward in order to get around such integrals [16]-[20]. In this paper, we opt to test the EFIE over volumetric subdomains attached to the boundary surface, inside the body under analysis, so that all the contour integrals can be evaluated numerically [17],[18]. In general, the MFIE, unlike the EFIE, is amenable to a wide range of discretization schemes. The RWG-discretization of the MFIE [21], though, exhibits poor accuracy [22]. Other MoM-implementations of the MFIE, with Galerkin testing - divergence-conforming [23],[24], curl-conforming [25],[26]) discontinuous (across edges) [9],[27] - , or conforming with the dual space of the operator range [28], have shown to mitigate such discrepancies to some extent. Some of these MFIE-schemes allow for the straightforward management of non-conformal meshes [29] or for mixed triangular-quadrangular tessellations [30].

The MFIE contributions in the discretization of the CFIE have been constrained to rely on divergenceconforming sets [23],[24],[31],[32] for the sake of compatibility with the EFIE entries. In consequence, the conventional RWG-discretization of the CFIE suffers from some loss of accuracy with respect to the EFIE and, more importantly, hampers the management of complex meshes. Conversely, the resulting linear systems show better condition numbers than their EFIE counterparts because of the well-conditioned MFIE matrix contributions. Over the last decade, other divergence-conforming implementations of the CFIE have shown improved accuracy with respect to the RWG choice [23],[24],[32]. Similarly, the so-called Discontinuous-Galerkin CFIE-based schemes [16], which adopt monopolar-RWG basis functions, allow for the analysis of some types of non-conformal meshes. However, the former are restricted to geometrically conformal triangulations and the latter do not report improved accuracy (versus the number of unknowns).

The monopolar-RWG implementations of the EFIE [17], [18], with volumetric testing, and the MFIE [9],[27], 
with Galerkin testing, exhibit improved accuracy versus the number of unknowns with respect to their RWGcounterparts in the analysis of subwavelength conductors with geometric singularities. As the electrical dimensions of the sharp-edged objects rise, though, the accuracy gain observed cannot counterbalance the significant increase of unknowns. In this context, the hybrid RWG/monopolar-RWG EFIE or MFIE schemes [9][17] bring out a sound compromise between accuracy (versus the number of unknowns) and versatility. Whereas the former is achieved through the balanced discretization of the current (RWG over smoothly meshed regions of the surface boundary and monopolar-RWG at facets touching sharp-edges), the latter is accomplished by the discontinuous modelling of the current across non-conformal mesh transitions.

In this paper, we present a new scheme of discretization of the CFIE based on the expansion of the current with the monopolar-RWG set. Our CFIE-implementation combines the monopolar-RWG discretization of the MFIE [9], Galerkin tested, and of the EFIE [17],[18], volumetrically tested. This is a facet-based scheme that, as we show in the paper, exhibits improved accuracy in the analysis of sharp-edged moderately small conductors with respect to the RWG-discretization. In this work, we provide guidelines on the best performing implementations in terms of the heights of the volumetric subdomains (wedges or tetrahedral elements), as regards the observed accuracy and the speed of convergence. Furthermore, we show the adequacy of our hybrid RWG/monopolar-RWG implementations of the CFIE for both accuracy and versatility purposes, through the successful analysis, respectively, of polyhedral targets or complex objects partitioned into independently meshed domains. In addition, we disclose the robustness of our monopolar-RWG CFIE schemes in the analysis of defective meshes for targets with geometric singularities.

\section{Surface integral equations}

The scattering analysis of a conductor immersed in the free space when illuminated by an incident plane wave $\left(\boldsymbol{E}^{\text {inc }}, \boldsymbol{H}^{\text {inc }}\right)$ can be formulated equivalently, in light of the Love's equivalence principle [33], through the distribution of electric equivalent current $\boldsymbol{J}$ over the surface enclosing the body $\boldsymbol{S}$ and the fulfillment of the electric-field or magnetic-field boundary conditions. This gives rise to the EFIE and the MFIE, which can be written, respectively, as

$$
\begin{gathered}
\eta_{0} T(\boldsymbol{J})=\hat{n} \times \boldsymbol{E}^{i n c} \\
\frac{\boldsymbol{J}}{2}-K(\boldsymbol{J})=\hat{n} \times \boldsymbol{H}^{i n c}
\end{gathered}
$$

where $\hat{n}$ stands for the unit normal vector pointing outwards and $\eta_{0}$ denotes the free-space impedance. The integral operators $T$ and $K$ are defined as

$$
\begin{gathered}
T(\boldsymbol{J})=j k \hat{n} \times \iint_{S}\left(I+\frac{\nabla \nabla \cdot}{k^{2}}\right) G\left(\boldsymbol{r}, \boldsymbol{r}^{\prime}\right) \cdot \boldsymbol{J} d s^{\prime} \\
K(\boldsymbol{J})=\hat{n} \times \iint_{S, C P V} \nabla G \times \boldsymbol{J} d s^{\prime}
\end{gathered}
$$

where $I$ stands for the identity operator and $k$ denotes the wavenumber. In (4), the integral is evaluated in the Cauchy principal value sense if the field point is on the surface of the target and $G\left(\boldsymbol{r}, \boldsymbol{r}^{\prime}\right)$ stands for the free-space Green's function, which is defined in phasor notation and with the $e^{+j \omega t}$ convention as

$$
G\left(\boldsymbol{r}, \boldsymbol{r}^{\prime}\right)=\frac{e^{-j k\left|\boldsymbol{r}-\boldsymbol{r}^{\prime}\right|}}{4 \pi\left|\boldsymbol{r}-\boldsymbol{r}^{\prime}\right|}
$$

The CFIE results from the weighted linear combination of the EFIE and the MFIE. In general, the combination factor $\alpha$ is set in order to make the resulting formulation either prone to the well-conditioned MFIE ( $\alpha$ close to 0$)$ or to the accurate EFIE ( $\alpha$ close to 1) [23],[32]. Our CFIE implementations provide the same relevance to both EFIE and MFIE contributions ( $\alpha=0.5)$, so that 


$$
\left[\hat{n} \times T+\frac{I}{2}-K\right]\left(\eta_{0} \boldsymbol{J}\right)=\hat{n} \times \hat{n} \times \boldsymbol{E}^{i n c}+\eta_{0} \hat{n} \times \boldsymbol{H}^{i n c}
$$

where, as is customary, the MFIE contribution is $\eta$-normalized so that the addition of the electric and magnetic contributions becomes numerically compatible.

\section{Facet-based discretization:}

The monopolar-RWG basis functions define three basis functions at each facet of the triangulation. Each basis function grows linearly from each vertex towards the opposed edge, which corresponds with the same local definition of the RWG basis functions but with no inter-facet continuity constraints across edges. Hence, for closed meshes, the number of unknowns amounts to three times the number of triangular facets $\left(N_{t}\right)$. Equivalently, in conformal triangulations, the monopolar-RWG expansion gives rise to two independent unknowns at both sides of each edge, which doubles the number of unknowns with respect to the RWG expansion.

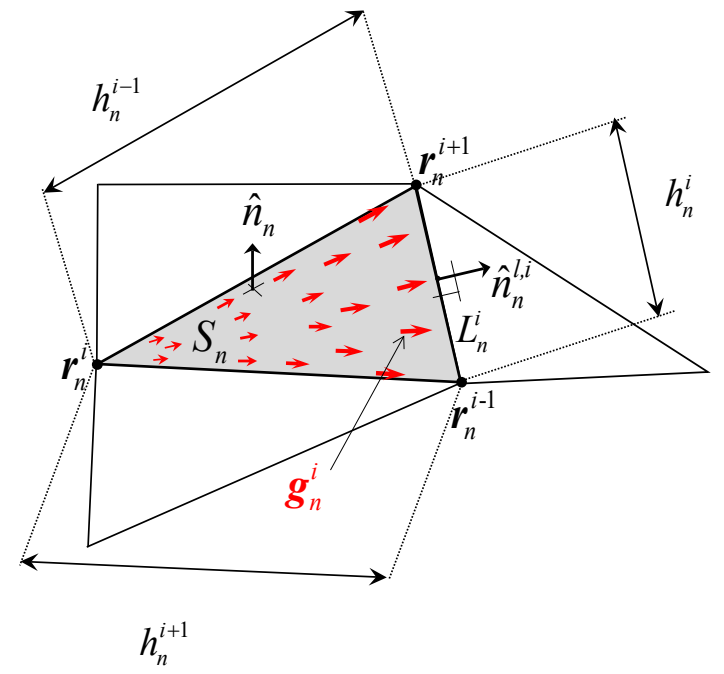

Fig. 1 - Monopolar-RWG basis function associated with the $i$ th local vertex of the $n$th triangle $S_{n}$. The index arithmetic is performed modulo three.

The monopolar-RWG basis function associated with the $p$ th vertex of the $n$th triangular facet is defined as

$$
\boldsymbol{g}_{n}^{p}\left(\boldsymbol{r}^{\prime}\right)=\frac{1}{2 A_{n}}\left(\boldsymbol{r}^{\prime}-\boldsymbol{r}_{n}^{p}\right) \quad \boldsymbol{r}^{\prime} \in S_{n}, \quad p=1,2,3
$$

where $\boldsymbol{r}_{n}^{p}$ denotes the position vector of the $p$-th vertex of the source triangle $S_{n}$ (see Fig. 1), with area $A_{n}$. In general, the discretization of the electric current in terms of the set of monopolar-RWG basis functions yields

$$
\boldsymbol{J}\left(\boldsymbol{r}^{\prime}\right) \simeq \sum_{n=1}^{N_{t}} \sum_{p=1}^{3} c_{n}^{p} \boldsymbol{g}_{n}^{p}\left(\boldsymbol{r}^{\prime}\right)
$$

where $\left\{c_{n}^{p}\right\}=\left\{c_{1}^{1} \ldots c_{N_{t}}^{1}, c_{1}^{2} \ldots c_{N_{t}}^{2}, c_{1}^{3} \ldots c_{N_{t}}^{3}\right\}$ denotes the set of unknown coefficients in the resulting matrix system. 


\subsection{MFIE}

The MoM-discretization of the MFIE with the monopolar-RWG basis functions and Galerkin testing involves the expansion of the current and the testing of the magnetic field at the boundary surface with the same monopolarRWG set, which leads to the following matrix system

$$
H_{\mathrm{inc}}^{[q, i]}=\sum_{n=1}^{N_{t}} \sum_{p=1}^{3} \mathrm{Z}_{[n, p]}^{H,[q, i]} c_{n}^{p} \quad 1 \leq q \leq N_{t}, 1 \leq i \leq 3
$$

with the excitation vector entry $H_{\text {inc }}^{[q, i]}$ and the impedance elements $Z_{[n, p]}^{H,[q, i]}$ defined as [21]

$$
\begin{gathered}
H_{\mathrm{inc}}^{[q, i]}=\iint_{S_{q}} \boldsymbol{g}_{q}^{i} \cdot \hat{n}_{q} \times \boldsymbol{H}^{i n c} d s \\
Z_{[n, p]}^{H,[q, i]}=\frac{1}{2} \iint_{S_{q}} \boldsymbol{g}_{q}^{i} \cdot \boldsymbol{g}_{n}^{p} d s-\iint_{S_{q}} \boldsymbol{g}_{q}^{i} \times \hat{n}_{q} \cdot \iint_{S_{n}, C P V} \nabla G \times \boldsymbol{g}_{n}^{p} d s^{\prime} d s
\end{gathered}
$$

where $S_{n}$ denotes the source triangle and $\hat{n}_{q}$ stands for the unit vector perpendicular to the testing facet $S_{q}$ pointing outwards.

\subsection{EFIE}

The discretization of the EFIE with the monopolar-RWG basis functions, discontinuous at edges, and Galerkin testing gives rise to hypersingular kernel contributions. The integration by parts of these double gradient kernel contributions leads to contour-surface or double-surface integrals, easy-to-manage through well-known integration routines, and to double-contour integrals, which become unbounded for self or edge-adjacent interactions. Recently, several non-Galerkin approaches [17]-[19] have successfully managed such contributions by shifting the testing subdomains off the boundary-surface, inside the body under analysis, where the electric field must be zero too. The fields are then tested over small volumetric entities (tetrahedral elements [17] or wedges [18]) attached to the boundary triangulation.

The monopolar-RWG discretization of the EFIE with volumetric testing gives rise to the following matrix system

$$
E_{\text {inc }}^{[q, i]}=\eta_{0} \sum_{n=1}^{N_{t}} \sum_{p=1}^{3} Z_{[n, p]}^{E[[, i]} c_{n}^{p} \quad 1 \leq q \leq N_{t}, 1 \leq i \leq 3
$$

and the excitation vector $E_{\text {inc }}^{[q, i]}$ is defined as follows

$$
E_{\mathrm{inc}}^{[q, i]}=\iiint_{V_{q}^{i}} \boldsymbol{T}_{q}^{i}(\boldsymbol{r}) \cdot \boldsymbol{E}^{i n c} d v
$$

where $\boldsymbol{T}_{q}^{1}, \boldsymbol{T}_{q}^{2}, \boldsymbol{T}_{q}^{3}$ stand for the three testing functions associated with the $q$-th facet of the boundary triangulation, $S_{q}$. Whereas for the tetrahedral testing three different subdomains, respectively, $V_{q}^{1}, V_{q}^{2}, V_{q}^{3}$, are defined (see Fig. 2), for the wedge testing the three testing functions share the same subdomain, whereby $V_{q}^{1}=V_{q}^{2}=V_{q}^{3}=V_{q}$ (see Fig. 3). 


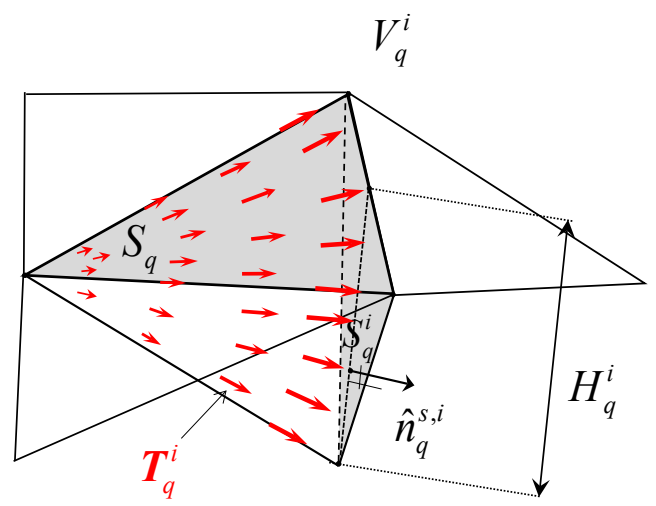

Fig. 2 - Testing function defined over the tetrahedral subdomain $V_{q}^{i}$, attached to the surface triangle $S_{q}$ and associated with the $i$ th local vertex. The volumetric subdomain $V_{q}^{i}$ is located inside the body under analysis.

The impedance elements in (13) $Z_{[n, p]}^{E,[q, i]}$ are defined as [17],[18]

$$
\begin{aligned}
Z_{[n, p]}^{E[, q, i]} & =j k \iiint_{V_{q}^{\prime}} \boldsymbol{T}_{q}^{i}(\boldsymbol{r}) \cdot \iint_{S_{n}} G\left(\boldsymbol{r}, \boldsymbol{r}^{\prime}\right) \boldsymbol{g}_{n}^{p}\left(\boldsymbol{r}^{\prime}\right) d s^{\prime} d v \\
& +j k^{-1} \iint_{S_{q}^{\prime}}\left(\boldsymbol{T}_{q}^{i}(\boldsymbol{r}) \cdot \hat{n}_{q}^{s, i}\right) \iint_{S_{n}} G\left(\boldsymbol{r}, \boldsymbol{r}^{\prime}\right) \nabla^{\prime} \cdot \boldsymbol{g}_{n}^{p}\left(\boldsymbol{r}^{\prime}\right) d s^{\prime} d s \\
& -j k^{-1} \iiint_{V_{q}^{\prime}} \nabla \cdot \boldsymbol{T}_{q}^{i}(\boldsymbol{r}) \iint_{S_{n}} G\left(\boldsymbol{r}, \boldsymbol{r}^{\prime}\right) \nabla \nabla^{\prime} \cdot \boldsymbol{g}_{n}^{p}\left(\boldsymbol{r}^{\prime}\right) d s^{\prime} d v \\
& -j k^{-1} \iint_{S_{q}^{i}}\left(\boldsymbol{T}_{q}^{i}(\boldsymbol{r}) \cdot \hat{n}_{q}^{s, i}\right) \iint_{L_{n}^{p}} G\left(\boldsymbol{r}, \boldsymbol{r}^{\prime}\right)\left(\boldsymbol{g}_{n}^{p}\left(\boldsymbol{r}^{\prime}\right) \cdot \hat{n}_{n}^{l, p}\right) d l^{\prime} d s \\
& +j k^{-1} \iiint_{V_{q}^{\prime}} \nabla \cdot \boldsymbol{T}_{q}^{i}(\boldsymbol{r}) \int_{L_{n}^{p}} G\left(\boldsymbol{r}, \boldsymbol{r}^{\prime}\right)\left(\boldsymbol{g}_{n}^{p}\left(\boldsymbol{r}^{\prime}\right) \cdot \hat{n}_{n}^{l, p}\right) d l^{\prime} d v
\end{aligned}
$$

where $L_{n}^{p}$ denotes the segment opposed to the $p$-th vertex of $S_{n}$ (see Fig. 1). In light of Figs. 2 and 3, $S_{q}^{i}$ represents the only side face of the volumetric subdomain where the normal component of the testing function $\boldsymbol{T}_{q}^{i}$ is nonzero. This face may become an isosceles triangle or a trapezoid depending on whether the testing is carried out with tetrahedral or wedge elements, respectively. The unit vectors $\hat{n}_{n}^{l, p}$ and $\hat{n}_{q}^{s, i}$ are normally pointing outwards through $L_{n}^{p}$ and $S_{q}^{i}$. The source contour integrals in (14) appear because the adopted monopolar-RWG expansion is discontinuous across edges.

The definition of the three testing functions straddling the three tetrahedral subdomains matching $S_{q}$ is [17]

$$
\boldsymbol{T}_{q}^{i}(\boldsymbol{r})=\frac{1}{2 A_{q} H_{q}^{i}}\left(\boldsymbol{r}-\boldsymbol{r}_{q}^{i}\right), \quad \boldsymbol{r} \in V_{q}^{i}, 1 \leq i \leq 3
$$

where $A_{q}$ and $H_{q}^{i}$ represent, respectively, the area of $S_{q}$ and the height of the testing tetrahedral element $V_{q}^{i}$ (see Fig. 2). We call the testing functions in (15) monopolar-SWG because, in an analogous manner as the monopolarRWG set, they arise from the divergence-conforming SWG set [34] but breaking the inter-element continuity requirement [17]. Similarly, the three testing functions straddling the testing wedge element $V_{q}$ are defined as [18]

$$
\begin{aligned}
\boldsymbol{T}_{q}^{i}(\rho, z)= & \frac{1}{2 A_{q} H_{q}}\left(\boldsymbol{\rho}_{q}^{i}-z \tan \alpha_{q}^{i} \hat{u}_{q}^{i}\right) \\
& \boldsymbol{r} \in V_{q}, 1 \leq i \leq 3,0 \leq z \leq H_{q}
\end{aligned}
$$


where $H_{q}$ represents the height of the testing wedge element $V_{q}$ and the vector $\boldsymbol{\rho}_{q}^{i}$ represents the radial position vector of the observation point associated with the $i$-th vertex (see Fig. 3). The variable $z$ denotes the distance from $S_{q}$ to the observation point. Note that the so-called wedge-monopolar function in (16) is linearly growing and becomes zero along the edge of the wedge $V_{q}$ meeting the $i$-th vertex [18]. The angle $\alpha_{q}^{i}$ and the unit vector $\hat{u}_{q}^{i}$, parallel to $S_{q}$, are defined in [18] (see Fig. 3).

In all these numerical implementations, the heights of the testing elements $(H)$ are set in terms of the mesh parameter $(h)$, which stands for the length of the edges arising from the discretization. As a matter of fact, these EFIE-implementations show improved accuracy with a proper choice of $H$ [17]-[19]. Whereas in the tetrahedral testing $H_{q}^{i}$ is defined as a fraction of $h_{q}^{i}$, the length of the edge opposed to the $i$-th surface vertex [17], in the wedge testing, $H_{q}$ is set as a fraction of the average of the lengths of the edges of $S_{q}, \sum_{i=1}^{3} h_{q}^{i} / 3$ [18]. The testing subdomains at the $q$-th facet are defined conformal to the boundary when the side faces of the volumetric testing elements lie over planes bisecting the angles formed by $S_{q}$ and the adjacent triangles. Obviously, this scheme is only valid for conformal meshes because of the assumed existence of a single edge between $S_{q}$ and each of the adjacent facets. Although the definition conformal-to-the-boundary enables the testing elements to follow the shape of the surface triangulation, a previous task of search and identification of adjacent facets around each facet of the meshing is required. Clearly, such search task is doomed to fail in non-conformal or defective meshes because of the presence of pairs of adjacent facets without a single edge in common or with slits in between.

Another definition of the volumetric testing subdomains, non-conformal-to-the-boundary, simplifies the construction of the testing functions: (a) the opposed face of the tetrahedral elements is oriented normally with respect to the surface facets; (b) the wedges become right triangular prisms. This scheme depends exclusively on the corresponding surface facet involved, with no need to check the facets around, which facilitates the manipulation of any kind of mesh, conformal, non-conformal or defective. Furthermore, the numerical implementations become somewhat easier; for example, in the computation of the impedance elements $\hat{n}_{q}^{s, i}=\hat{n}_{q}^{l, i}$ or the wedge testing through right triangular prisms enforces $\alpha_{q}^{i}=0$ in (16) [18]. For facets near abrupt convex geometrical singularities, though, these subdomains are prone to cross the boundary surface. This may give rise to some error that can be minimized through the sufficient reduction of the height of the testing entities [18].

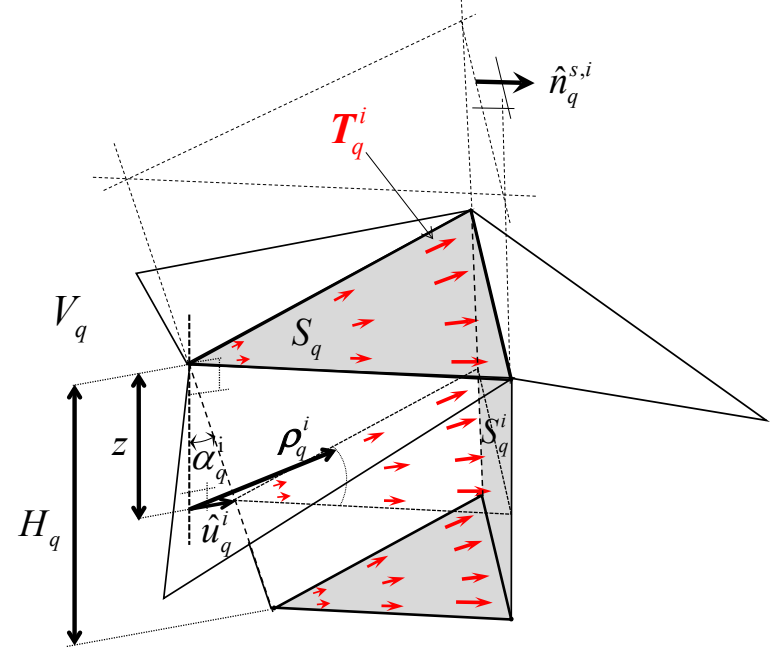

Fig. 3 - Testing function defined over the wedge subdomain $V_{q}$, attached to the surface triangle $S_{q}$ and associated with the $i$ th local vertex. The volumetric subdomain $V_{q}$ is located inside the body under analysis. 


\subsection{CFIE}

Our monopolar-RWG discretization of the CFIE results from the combination of the Galerkin monopolar-RWG implementation of the MFIE [9] and the discontinuous implementations of the EFIE with volumetric testing [17],[18]. The discretization of the MFIE with surface testing and monopolar-RWG basis functions is valid in numerical terms and, additionally, shows improved accuracy in the scattering analysis of sharp-edged conductors with respect to the conventional RWG-discretization [9], [27]. In this paper, we disregard monopolar-RWG implementations of the CFIE relying on MFIE-schemes that make use of the off-boundary testing because our tests show no benefit in accuracy terms when compared with the standard Galerkin monopolar-RWG discretization [35]. Our monopolar-RWG discretization of the CFIE is thus based on a surface-volumetric testing scheme, which gives rise to the following matrix system

$$
E H_{\mathrm{inc}}^{[q, i]}=\sum_{n=1}^{N_{t}} \sum_{p=1}^{3} \mathrm{Z}_{[n, p]}^{C,[, i]} c_{n}^{p} \quad 1 \leq q \leq N_{t}, 1 \leq i \leq 3
$$

In light of (6), the excitation vector and the impedance elements above are defined as

$$
\begin{gathered}
E H_{\mathrm{inc}}^{[q, i]}=E_{\mathrm{inc}}^{[q, i]}+\eta_{0} H_{\mathrm{inc}}^{[q, i]} \\
\mathrm{Z}_{[n, p]}^{C,[q, i]}=\eta_{0}\left(\mathrm{Z}_{[n, p]}^{E[[q, i]}+\mathrm{Z}_{[n, p]}^{H,[q, i]}\right)
\end{gathered}
$$

\section{Hybrid discretization:}

The adoption of facet-based schemes for accuracy purposes becomes justified as long as they manifest improved accuracy with respect to the RWG schemes and same number of unknowns. Since the schemes discontinuous (across edges) involve more computational burden for a given mesh than the RWG approaches, which handle half the number of unknowns, the monopolar-RWG schemes are constrained to the adoption of coarser meshes in the accuracy tests. In general, the improved accuracy observed for the monopolar-RWG schemes with respect to the RWG choice depends on the relevance of the sharp-edge contributions in the scattering pattern. Indeed, monopolarRWG implementations of the EFIE [17]-[19] or the MFIE [9],[27], for conductors, and of the PMCHWT formulation, for dielectric objects with moderate or high contrast [11],[12] or for ferromagnetic targets [36], exhibit improved accuracy in the scattering analysis of subwavelength sharp-edged targets. For growing electrical dimensions, though, the scattering contribution associated with the sharp edges of the object becomes decreasingly relevant and the accuracy gain observed for the facet-based schemes diminishes. Clearly, for big enough electrical dimensions, in much the same way as for smooth targets, the improved observed accuracy does not compensate for the relevant increase of unknowns required. In this context, so-called hybrid RWG/monopolar-RWG schemes have shown similar accuracy for the EFIE and the MFIE as the fully monopolar-RWG implementations using many less unknowns [9],[17],[18].

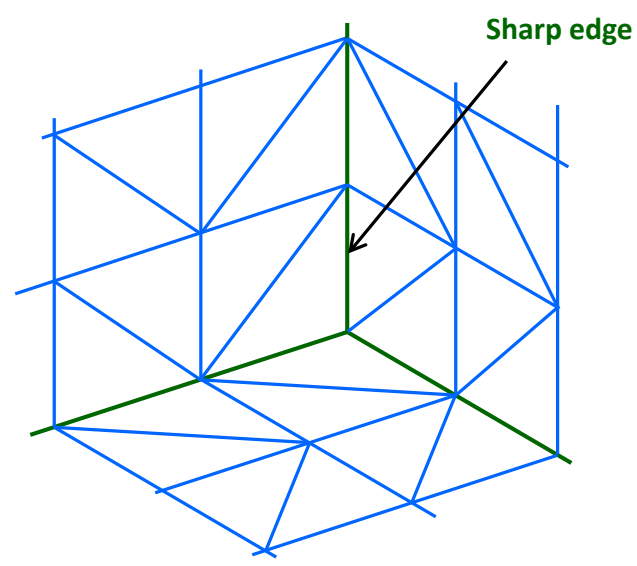

(a) 


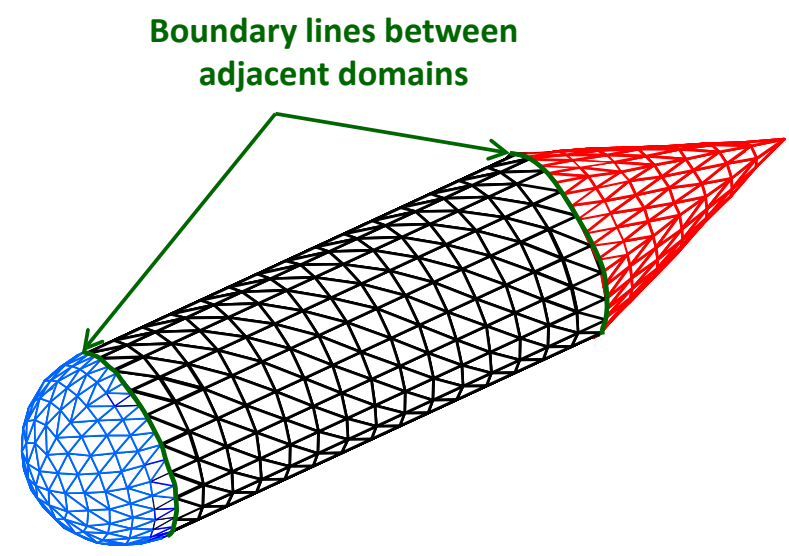

(b)

Fig. 4 - Hybrid Discretization over the boundary of a polyhedron: monopolar-RWG basis functions on facets touching (a) sharp edges or (b) lines between adjacent domains and RWG basis functions elsewhere.

The Domain Decomposition Methods (DDM) undertake the scattering analysis through the partition of the object into several domains. Each domain is amenable to a locally appropriate scheme of meshing, which is a proof of flexibility. However, the enforcement of continuity conditions across the boundaries of adjacent domains is normally required too. The DDM in [37] makes use of RWG basis functions but defines artificial currents over the intersecting boundaries in order to impose the continuity conditions across adjacent domains. This scheme allows the analysis of non-conformal meshes at the cost of increasing the number of unknowns. Facet-based (discontinuous across edges) schemes are better suited than edge-based RWG-methods for the agile and flexible analysis of complex meshes through the DDM. The discontinuous Galerkin schemes [16], which define monopolar-RWG basis functions strictly over facets touching the boundaries between contiguous domains [7], [38], give rise to an evident reduction on the number of unknowns. However, some so-called penalty conditions are still required to weakly enforce the continuity of the current at both sides of the separating boundaries. In general, the implementation of such penalty conditions is not trivial because of the required search for the intersecting edges between nonmatching meshes. Additionally, for intersecting contours with non-coplanar facets at both sides, with spurious slits arising between facets over the intersecting contour, the imposition of the continuity conditions becomes very troublesome (if not impossible) since there are no clear intersections [39].

In this paper, we develop a hybrid RWG/monopolar-RWG discretization of the CFIE for both accuracy and versatility purposes. This hybrid scheme, formerly presented in [9] for the MFIE, carries out the monopolar-RWG expansion (and volumetric electric-field testing) strictly through facet edges matching, respectively, sharp edges (see Fig. 4-(a)) or domain boundary lines (see Fig. 4-(b)) and defines the RWG expansion elsewhere. Another hybrid scheme, presented in [17] for the EFIE, relies on the decomposition of the monopolar-RWG contributions into two terms, normally continuous (even) or discontinuous (odd) across edges. This approach relies on the RWGdiscretization of the EFIE and is thereby restricted to conformal meshes. Although our tests show that the accuracy of both hybrid implementations for the CFIE is very similar, the former hybrid implementation, [hyb][monoR], provides in practice greater flexibility than the latter, [hyb][e-o-monoR], since it allows the management of nonconformal mesh transitions (see Fig. 4-(b)). In this paper, the accuracy tests in subsection 5.1 are carried out for sharp-edged conductors such that the mesh transitions at sharp-edges are geometrically conformal. On the other hand, the tests on versatility in subsection 5.4 assume nonconformal mesh transitions across the boundary lines of the partitioning domains. In both cases, CFIE[hyb][monoR] is implemented such that the monopolar-RWG basis functions (and electric-field volumetric testing) are defined accordingly over facets touching either sharp edges or domain boundary lines.

The hybrid expansion of the electric current yields:

$$
\boldsymbol{J}\left(\boldsymbol{r}^{\prime}\right) \simeq \sum_{n=1}^{N_{f}} c_{n} \boldsymbol{f}_{n}\left(\boldsymbol{r}^{\prime}\right)+\sum_{n=1}^{N_{g}} d_{n} \boldsymbol{g}_{n}\left(\boldsymbol{r}^{\prime}\right)
$$


where $\left\{\boldsymbol{f}_{n}\right\}$ and $\left\{\boldsymbol{g}_{n}\right\}$ stand for the sets of RWG and monopolar-RWG basis functions, respectively. The sets $\left\{c_{n}\right\},\left\{d_{n}\right\}$ denote the unknown coefficients associated, respectively, with each of these sets of basis functions. The normal component of the monopolar-RWG basis functions in (20) leaks out through a sharp edge (or a boundary domain line) in the corresponding facet (see Fig. 4). Whereas $N_{f}$ stands for the number of edges inside the faces of the polyhedral object (or inside the partitioning domains), $N_{g}$ denotes the overall number of facet sides that match sharp edges (or boundary domain lines) of the object. In general, the computational load with the hybrid discretization involves $N_{f}+N_{g}$ unknowns, much closer to the $N_{f}+0.5 N_{g}$ unknowns involved in the RWG choice than the $2 N_{f}+N_{g}$ unknowns required in the fully monopolar-RWG discretization. This becomes especially evident for targets with moderate or big electrical dimensions, where $N_{f}>N_{g}$.

In light of (24), the hybrid RWG/monopolar-RWG discretization of the CFIE results in the following matrix system

$$
\begin{aligned}
E H_{\mathrm{inc}}^{f, r} & =\sum_{\mathrm{n}=1}^{N_{f}} \mathrm{Z}_{r n}^{C, f f} c_{n}+\sum_{\mathrm{n}=1}^{N_{g}} \mathrm{Z}_{r n}^{C, f g} d_{n}, \quad r=1 . . N_{f} \\
E H_{\mathrm{inc}}^{g, q} & =\sum_{\mathrm{n}=1}^{N_{f}} \mathrm{Z}_{q n}^{C, g f} c_{n}+\sum_{\mathrm{n}=1}^{N_{g}} \mathrm{Z}_{q n}^{C, g g} d_{n}, \quad q=1 . . N_{g}
\end{aligned}
$$

where the excitation vector entries and the impedance elements result from the combinations in (18) and (19) of the electric-field and magnetic-field contributions, such that

$$
\begin{gathered}
E H_{\mathrm{inc}}^{x}=E_{\mathrm{inc}}^{x}+\eta_{0} H_{\mathrm{inc}}^{x} \\
\mathrm{Z}^{C, x y}=\eta_{0}\left(\mathrm{Z}^{E, x y}+\mathrm{Z}^{H, x y}\right)
\end{gathered}
$$

with the superscript $x$ denoting the adopted scheme of testing, either RWG $(f)$ or surface-volumetric $(g)$, as in (18) or (19). Likewise, $y$ refers to the adopted scheme of expansion of the current, RWG $(f)$ or monopolar-RWG $(g)$.

As regards the implementation of the magnetic-field impedance elements in (23), $Z^{H, x y}$, the procedure is formally the same as in (11) for the impedance submatrices in (21), except that the $f y$ (or $x f$ ) elements require the testing (or expansion) with RWG basis functions instead of monopolar-RWG. Computationally speaking, this is straightforward because the RWG scheme of MFIE testing (or expansion) result from the combination of the monopolar-RWG contributions defined over the pair of triangles sharing each edge.

The electric-field $f f$ or $g g$ impedance elements in (21) and (23) arise, respectively, from the RWG-Galerkin discretization or the volumetrically tested monopolar-RWG discretization, in (14), of the EFIE. The mathematical expression for the other electric-field impedance entries, $f g$ or $g f$, becomes

$$
\begin{aligned}
Z_{r n}^{E, f g} & =j k \iint_{S_{r}^{\prime} \cup S_{r}^{2}} \boldsymbol{f}_{p}(\boldsymbol{r}) \cdot \iint_{S_{n}} G\left(\boldsymbol{r}, \boldsymbol{r}^{\prime}\right) \boldsymbol{g}_{n}\left(\boldsymbol{r}^{\prime}\right) d s^{\prime} d s \\
& -j k^{-1} \iint_{S_{r}^{\prime} \cup S_{r}^{2}} \nabla \cdot \boldsymbol{f}_{p}(\boldsymbol{r}) \iint_{S_{n}} G\left(\boldsymbol{r}, \boldsymbol{r}^{\prime}\right) \nabla^{\prime} \cdot \boldsymbol{g}_{n}\left(\boldsymbol{r}^{\prime}\right) d s^{\prime} d s \\
& +j k^{-1} \iint_{S_{r}^{\prime} \cup S_{r}^{2}} \nabla \cdot \boldsymbol{f}_{p}(\boldsymbol{r}) \int_{L_{n}^{\prime(k)}} G\left(\boldsymbol{r}, \boldsymbol{r}^{\prime}\right)\left(\boldsymbol{g}_{n}\left(\boldsymbol{r}^{\prime}\right) \cdot \hat{n}_{n}^{s h(n)}\right) d l^{\prime} d s
\end{aligned}
$$

where $s h$ denotes the set of local triangle indices associated with the set of zero-current vertices or their opposed edges in the definition of the monopolar-RWG set $\left\{\boldsymbol{g}_{n}\right\}$ (see Fig. 1), and

$$
\begin{aligned}
& \mathrm{Z}_{q n^{E}}^{E, g f}=j k \iiint_{V_{q}^{\prime \prime(q)}} \boldsymbol{T}_{q}^{s h(q)}(\boldsymbol{r}) \cdot \iint_{S_{n}^{\prime} \cup S_{n}^{2}} G\left(\boldsymbol{r}, \boldsymbol{r}^{\prime}\right) \boldsymbol{f}_{n}\left(\boldsymbol{r}^{\prime}\right) d s^{\prime} d v \\
& +j k^{-1} \iint_{S_{q}^{(q)}}\left(\boldsymbol{T}_{q}^{s h(q)}(\boldsymbol{r}) \cdot \hat{n}_{q}^{s, s h(q)}\right) \iint_{S_{n}^{\prime} \cup S_{n}^{2}} G\left(\boldsymbol{r}, \boldsymbol{r}^{\prime}\right) \nabla^{\prime} \cdot \boldsymbol{f}_{n}\left(\boldsymbol{r}^{\prime}\right) d s^{\prime} d s \\
& -j k^{-1} \iiint_{V_{q}^{\prime \prime(q)}} \nabla \cdot \boldsymbol{T}_{q}^{s h(q)}(\boldsymbol{r}) \iint_{S_{n}^{\prime} \cup S_{n}^{2}} G\left(\boldsymbol{r}, \boldsymbol{r}^{\prime}\right) \nabla^{\prime} \cdot \boldsymbol{f}_{n}\left(\boldsymbol{r}^{\prime}\right) d s^{\prime} d v
\end{aligned}
$$


where the superscript $s h$ here includes the local indices that identify the volumetric subdomains or their opposed surfaces that define the volumetric testing functions (see Figs. 2, 3). The expressions in (24) and (25) derive from the fully facet-based expression in (14) after applying the normal continuity constraint across edges of the RWG basis functions in the testing scheme, in (24), or in the current expansion procedure, in (25).

\section{Results}

In subsection 5.1, we demonstrate that our facet-based CFIE implementation solves, as expected, the interiorresonance problem. Furthermore, we show results that illustrate the performance of our monopolar-RWG implementations of the CFIE as regards accuracy, speed of convergence, grid-robustness and versatility. In subsection 5.2, we show the accuracy of our monopolar-RWG implementations of the CFIE with respect to their RWG-counterparts. Moreover, we provide guidelines for the best performing range of heights $(H)$ of the testing volumetric elements. In subsection 5.3, we bring to light the speed of convergence of our CFIE-schemes. We show the range of $H$-values for which the reduction in the number of iterations to reach convergence with respect to the EFIE is most evident. In subsection 5.4, we address the suitability of our schemes for preserving the accuracy in some types of slightly defective meshes. We focus especially on meshes with misaligned vertices at sharp edges, for which, in our experience, our schemes are best suited. Finally, in subsection 5.5, we show results that exhibit the adequacy of our monopolar-RWG schemes for the successful analysis of structures partitioned into several domains and independently meshed with geometrically non-conformal meshes, where the traditional edge-based schemes fail.

In order to obtain a fair assessment of the observed performance, we minimize the sources of error in the numerical implementations. In the accuracy tests, for example, we obtain the direct solution of the resulting matrix system through the Gaussian Elimination procedure (subsections 5.2, 5.4 and 5.5). In addition, we carry out the surface or volumetric testing schemes of the incident fields with, respectively, three-point or five-point Gaussian rules. Moreover, we compute the RCS with a three-point quadrature rule. Similarly, the accurate computation of the impedance elements embraces the following measures:

- Singularity subtraction of the high-order kernel contributions in the inner surface or volumetric integrals [40] for all the interactions involved.

- Numerical evaluation of the low-order kernel contributions with Gaussian numerical rules ( 9 quadrature points for the surface inner integrals [41] and 11 cubature points for the tetrahedral inner integrals [42]).

- Computation of the volumetric integrals over wedges, conformal-to-the-boundary, through the decomposition of each wedge into three tetrahedral elements. As for the right triangular prisms arising from the wedge testing, nonconformal-to-the-boundary, we apply 6-point quadrature schemes (resulting from the allocation of two legendrian points over the normal direction to the boundary and three quadrature points over the parallel direction).

- Computation of the outer line or surface integrals with a 9-point quadrature rule and of the outer volumetric integrals with a cubature rule of 11 points

- Swap between the inner line-integrals and the outer surface or volumetric integrals in the monopolar-RWG implementations with tetrahedral testing or with wedge testing non-conformal-to-the-boundary.

In the graphs below, we name the conventional Galerkin RWG-discretization of the EFIE, the MFIE and the CFIE, respectively as EFIE[R] [5], MFIE[R] [21] and CFIE[R]. Similarly, MFIE[monoR] denotes the Galerkin monopolar-RWG discretization of the MFIE [9]. We call the volumetrically tested monopolar-RWG implementations of the EFIE as EFIE[tet][monoR] or EFIE[wed][monoR], respectively, with tetrahedral or wedge elements [17],[18]. The implementations CFIE[tet][monoR] or CFIE[wed][monoR] result from the linear combination of EFIE[tet][monoR] or EFIE[wed][monoR], respectively, and MFIE[monoR]. Furthermore, we show results for the magnetic-field or electric-field hybrid formulations, which we call MFIE[hyb][R-monoR] [9] and EFIE[hyb][tet][R-monoR] [17] or EFIE[hyb][wed][R-monoR] [18], respectively. Similarly, CFIE[hyb][tet][RmonoR] or CFIE[hyb][wed][R-monoR], arise from the combination of MFIE[hyb][R-monoR] and, respectively, EFIE[hyb][tet][R-monoR] or EFIE[hyb][wed][R-monoR]. The adopted wavelength for all tested cases is $\lambda=1 \mathrm{~m}$. Whereas the volumetric elements in the fully monopolar-RWG or hybrid RWG/monopolar-RWG implementations in subsections 5.2 and 5.3 are defined conformal to the boundary, in subsections 5.4 and 5.5 they are set nonconformal to the boundary. 


\subsection{Interior-resonance}

Figs. 5 and 6 exhibit the continuous evolution over frequency of the computed backscattered RCS and the conditioning of the impedance matrices, respectively, of our monopolar-RWG discretization of the CFIE with tetrahedral testing. This confirms the success of our CFIE-implementations in circumventing the numerical anomalies observed at certain frequencies with the fundamental formulations EFIE[tet][monoR] or MFIE[monoR]. In particular, around these frequencies the condition number of the impedance matrix resulting from EFIE[tet][monoR] grows considerably (see Fig. 5) and the accuracy of the RCS computed with MFIE[monoR] deteriorates (see Fig. 6).

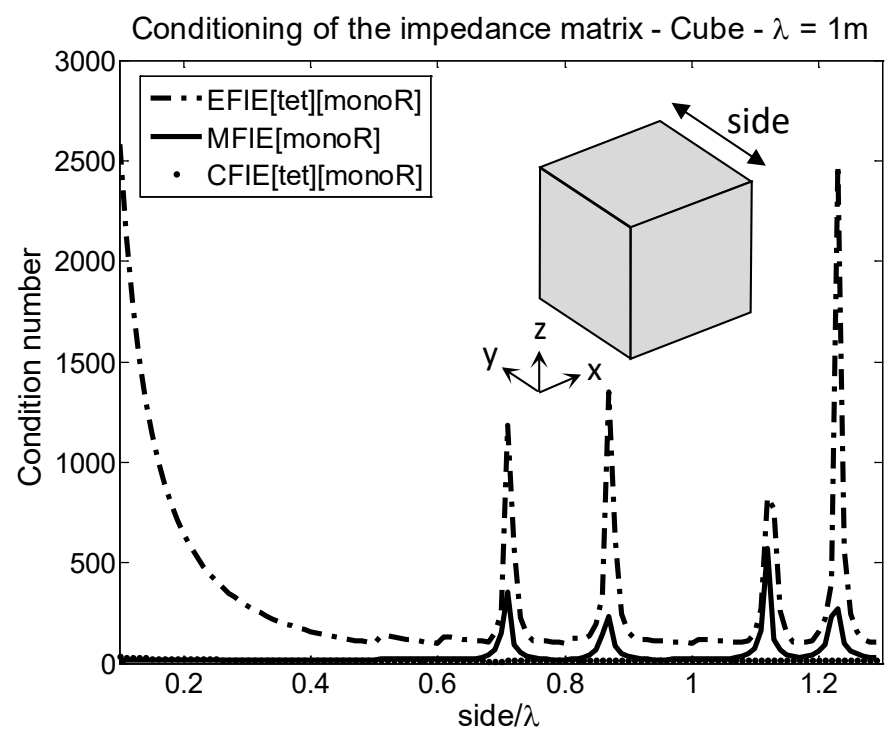

Fig. 5 - Condition number of the impedance matrix resulting from the facet-based schemes EFIE[tet][monoR], MFIE[monoR] and CFIE[tet][monoR] for a cube with growing electrical dimensions. For side dimensions above $0.5 \lambda$, the triangulations are defined with $h$ around $0.1 \lambda$. The adopted number of triangles evolves from $N t=300$ (if side $=0.5 \lambda$ ) to $N t=2028$ (if side $=1.3 \lambda$ ). For side dimensions below $0.5 \lambda$, $N t$ is set to 300 .

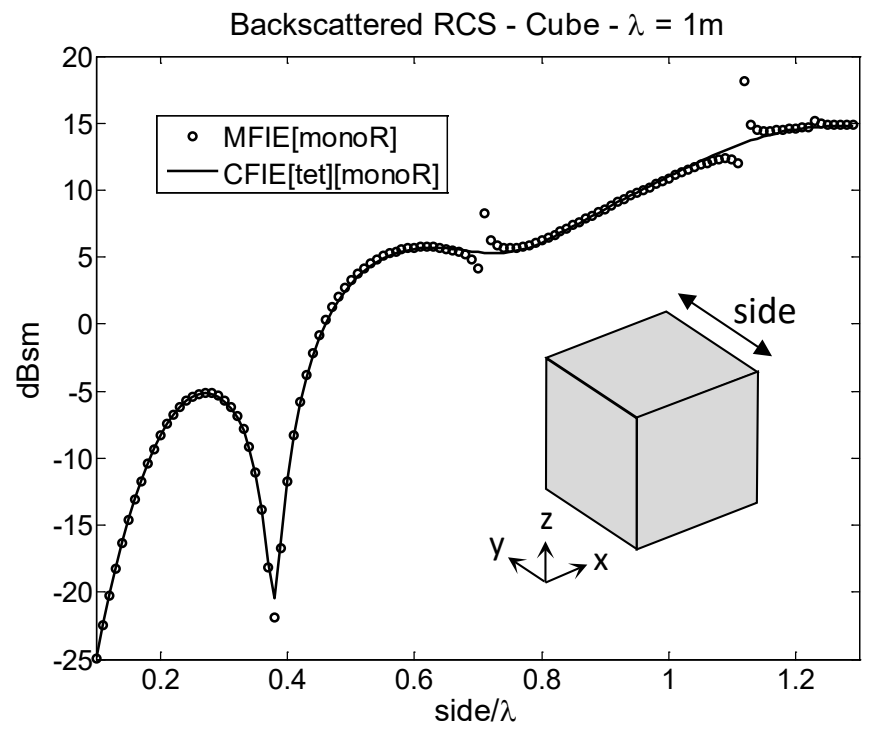

Fig. 6 - Backscattered RCS computed with the fully facet-based schemes MFIE[monoR] and CFIE[tet][monoR] for a cube with growing electrical dimensions and an impinging x-polarized z-propagating wave. For side dimensions above $0.5 \lambda$, the triangulations are defined with $h$ around $0.1 \lambda$, whereby the number of triangles evolves from $N t=300$ (if side $=0.5 \lambda$ ) to $N t=2028$ (if side $=1.3 \lambda$ ). For side dimensions below $0.5 \lambda$, $N t$ is set to 300 . 
The use of the CFIE appears justified in frequency regions with interior resonances, which involves big enough electrical dimensions. The full-wave CFIE-analysis may be extended to smaller electrical dimensions, thereby benefitting from the good balance between accuracy and conditioning (see Fig. 5). However, the extension as fullwave approach of the conventional MoM-CFIE schemes to the very low or extremely low frequency regimes is not easily workable since the corresponding EFIE or MFIE schemes from which the CFIE derives give rise to incorrect solutions because of current cancellations [43], [44], [45], [46].

\subsection{Accuracy}

In Figs. 7-14, we show RCS results under an impinging x-polarized z-propagating wave for our monopolar-RWG implementations of the CFIE, which we compare against the standard EFIE[R], MFIE[R], CFIE[R]. Moreover, we assess the accuracy of our CFIE-implementations discontinuous (across edges) with respect to their electric-field or magnetic-field counterparts [9],[17],[18]. We show results also for the recently developed CFIE-scheme in [24], which stems from adjusting the identity operator contribution in CFIE[R]. This scheme, which we name CFIE[R][id], shows improved accuracy with respect to the standard CFIE[R] [24].

We test the RCS-accuracy of our monopolar-RWG CFIE-implementations through the following root-meansquare relative error over a set of $M$ observation angles in the $\mathrm{E}$ and $\mathrm{H}$ planes [11],[12] as

$$
e_{f a r}=\frac{\left[\sum_{j=1}^{M}\left|R C S_{E}\left(\theta_{j}\right)-R C S_{E}^{R E F}\left(\theta_{j}\right)\right|^{2}+\sum_{j=2}^{M-1}\left|R C S_{H}\left(\theta_{j}\right)-R C S_{H}^{R E F}\left(\theta_{j}\right)\right|^{2}\right]^{1 / 2}}{\left[\sum_{j=1}^{M}\left|R C S_{E}^{R E F}\left(\theta_{j}\right)\right|^{2}+\sum_{j=2}^{M-1}\left|R C S_{H}^{R E F}\left(\theta_{j}\right)\right|^{2}\right]^{1 / 2}}
$$

where $\theta_{1}=0$ and $\theta_{M}=\pi-\pi / M[11],[12]$. We assess the errors with respect to benchmark results (REF) obtained with the conventional RWG-implementation of the EFIE and very fine unstructured mesh (around 45000 triangular facets per target) with $h$-refinement near sharp edges and corners. In our numerical tests, we adopt $M=60$.

We display the accuracy of the RCS computed with our volumetrically tested CFIE-implementations with respect to the height $H$ of the testing elements (Figs. 7-10) and the number of unknowns $N$ (Figs. 11-14). In Figs. 7-10, the monopolar-RWG implementations and their hybrid forms are constrained to coarser meshes in order to manage similar number of unknowns as the RWG schemes (CFIE[R] and CFIE[R][id]). In Figs. 11-14, we set the $H$ values for our monopolar-RWG implementations with the optimal values observed in Figs. 7-10 for the CFIE or in [17],[18] for the EFIE. We test the following electrically small objects with geometric singularities: a cube with side $0.1 \lambda$ (Figs. 7 and 11), a (regular) square pyramid with side $0.1 \lambda$ (Figs. 8 and 12) and a (regular) tetrahedron with side $0.25 \lambda$ (Figs. 9 and 13). Figs. 10 and 14 display RCS results for a tetrahedron with side $1 \lambda$. Despite the moderate electrical dimensions, this object is sharp-edged enough for the monopolar-RWG EFIE-implementations, especially hybrid, to exhibit improved accuracy with respect to EFIE[R] [17],[18]. 


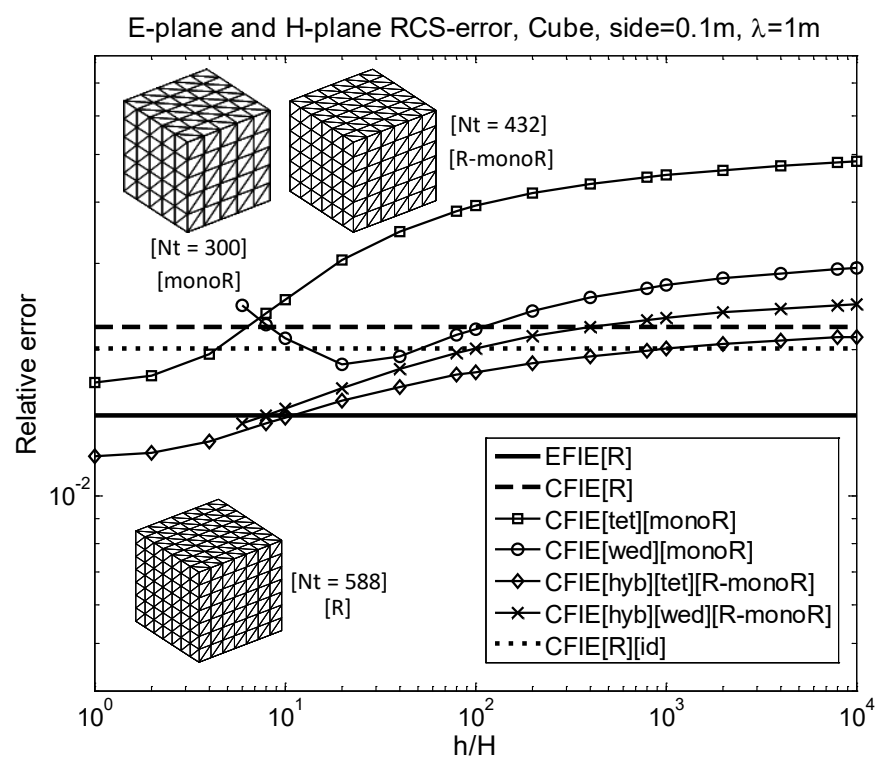

Fig. 7 - Relative RCS-error of our monopolar-RWG CFIE implementations, full or hybrid, with respect to CFIE[R] and EFIE[R] and similar number of unknowns, versus the height $H$ of the testing elements for a cube with side $0.1 \mathrm{~m}(\lambda=1 \mathrm{~m})$. Reference results computed with EFIE[R] and 71754 unknowns.

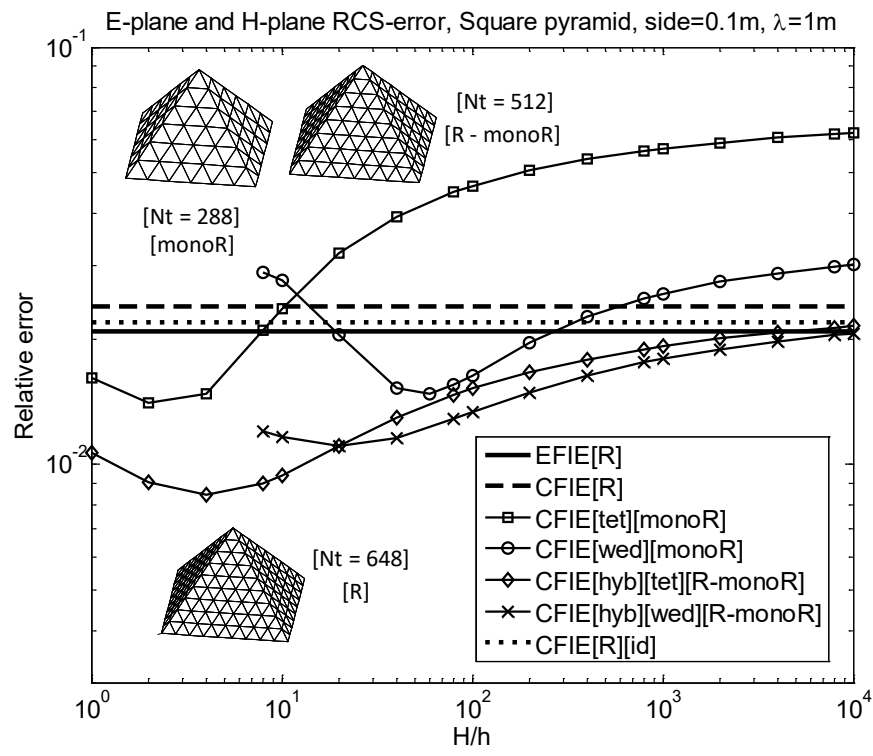

Fig. 8 - Relative RCS-error of our monopolar-RWG CFIE implementations, full or hybrid, with respect to CFIE[R] and EFIE[R] and similar number of unknowns, versus the height $H$ of the testing elements for a regular square pyramid with side $0.1 \mathrm{~m}(\lambda=1 \mathrm{~m})$. Reference results computed with EFIE[R] and 66918 unknowns.

In view of Figs. 7-9, the $H$-ranges of improved accuracy for our monopolar-RWG implementations of the CFIE with respect to the standard CFIE[R] and the optimized CFIE[R][id] are similar, though slightly narrower, than the analogous $H$-ranges for our monopolar-RWG EFIE-implementations [17],[18]. In particular, for the electrically small objects under analysis, the tetrahedral or wedge testing schemes show improved performance for $H$ ranges, respectively, roughly between $h / 10$ and $h$, or between $h / 500$ and $h / 10$. Interestingly, these nonconforming CFIEimplementations even show improved performance with respect to the standard EFIE[R]. This occurs for particularly narrow $H$-ranges and for the tetrahedron or the square-pyramid, with more pronounced sharp edges than the cube. In view of Figs. 7-9, the hybrid RWG/monopolar-RWG implementations of the CFIE, which define a discontinuous expansion only at edges touching the sharp edges, excel as very useful tools for the scattering analysis of electrically small sharp-edged objects because they provide similar accuracy as the fully discontinuous implementations but with many less unknowns. This demonstrates the adequacy of our monopolar-RWG implementations for the sound 
capture of the electromagnetic behavior associated with sharp edges. For a fixed number of unknowns, the observed relative error for the hybrid implementations then becomes much smaller than their RWG-counterparts and for very wide $H$-ranges, between $h / 10^{4}$ and $h$ roughly.

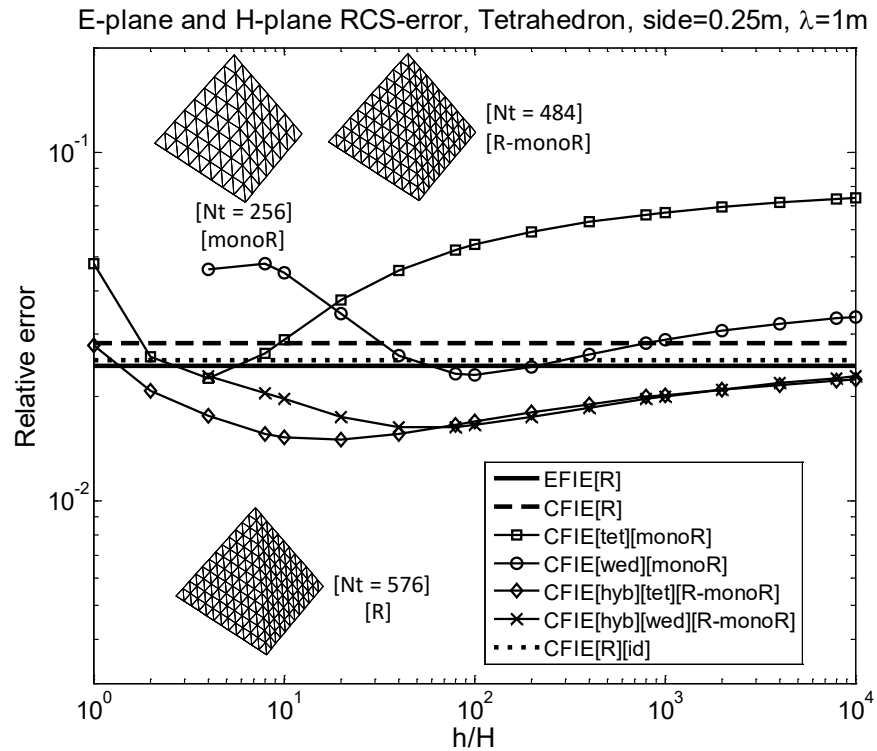

Fig. 9 - Relative RCS-error of our monopolar-RWG CFIE implementations, full or hybrid, with respect to CFIE[R] and EFIE[R] and similar number of unknowns, versus the height $H$ of the testing elements for a regular tetrahedron with side $0.25 \mathrm{~m}(\lambda=1 \mathrm{~m})$. Reference results computed with EFIE[R] and 68475 unknowns.

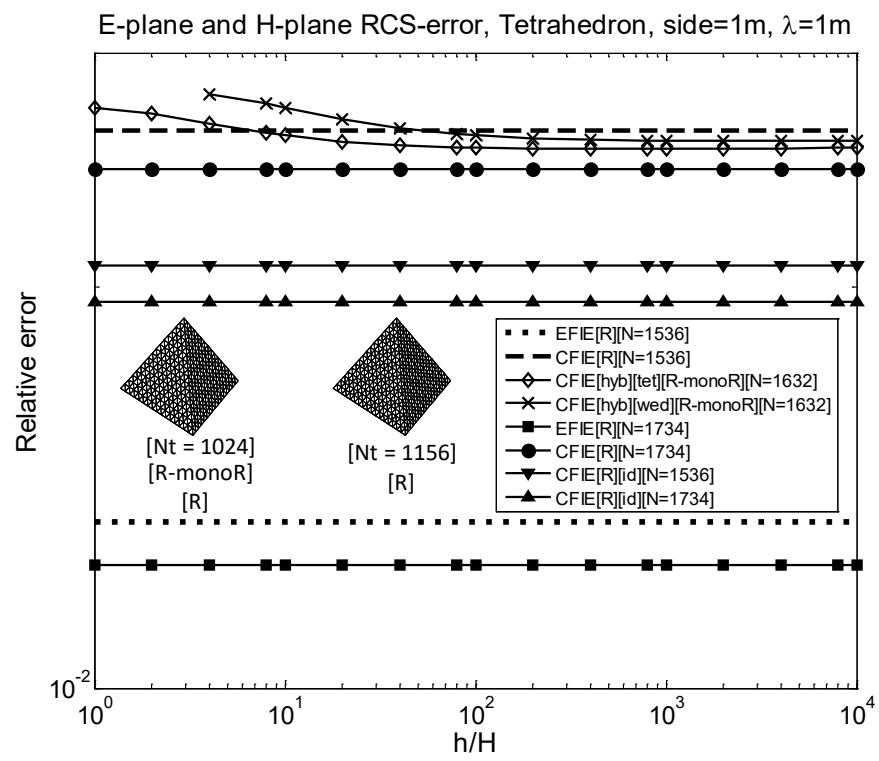

Fig. 10 - Relative RCS-error of our hybrid monopolar-RWG CFIE implementations, with respect to CFIE[R] and EFIE[R] and similar number of unknowns, versus the height $H$ of the testing elements for a regular tetrahedron with side $1 \mathrm{~m}(\lambda=1 \mathrm{~m})$. Reference results computed with EFIE[R] and 71121 unknowns.

In view of Figs. 11-13, as regards the electrically small targets, it is clear that the accuracy improvement versus $N$ as compared to the RWG-discretization is more evident in relative terms for the monopolar-RWG implementations of the EFIE or the MFIE, than for the CFIE. In any case, the improvement is still evident for the monopolar-RWG implementations of the CFIE, as compared to CFIE[R] and CFIE[R][id], especially for objects with pronounced sharp edges (tetrahedron or square pyramid). The hybrid RWG/monopolar-RWG CFIE-schemes, which maintain the 
observed accuracy of the monopolar-RWG CFIE-schemes with less unknowns, show clear enhanced performance with respect to $\mathrm{CFIE}[\mathrm{R}]$ and $\mathrm{CFIE}[\mathrm{R}][\mathrm{id}]$ for the three electrically small polyhedra.

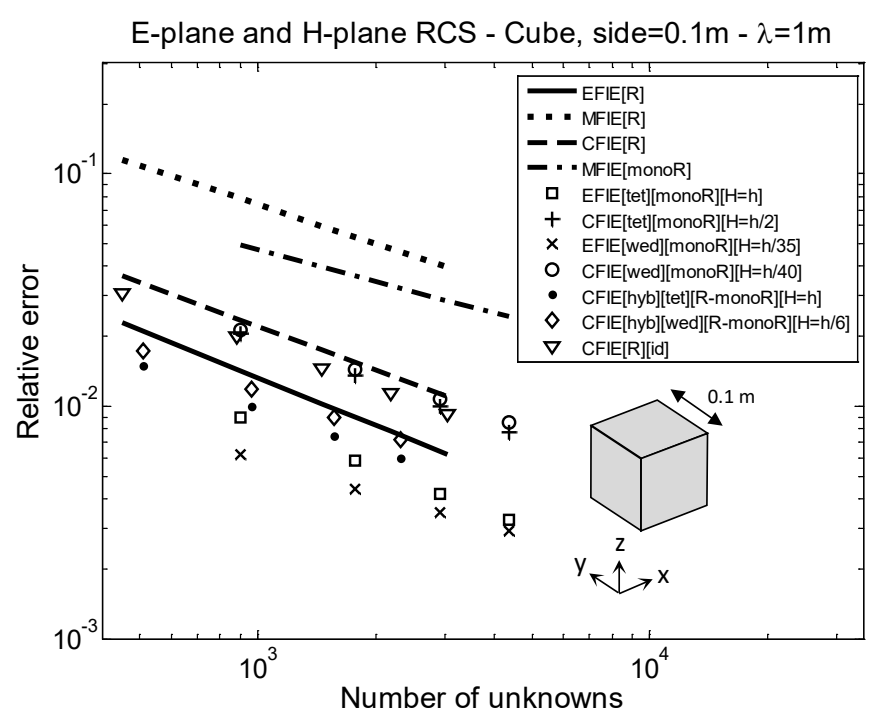

Fig. 11 - Relative RCS-error of several CFIE, EFIE or MFIE implementations, RWG, monopolar-RWG or hybrid RWG/monopolar-RWG, versus the number of unknowns for a cube with side $0.1 \mathrm{~m}(\lambda=1 \mathrm{~m})$. Reference results computed with EFIE[R] and 71754 unknowns.

For the $1 \lambda$-tetrahedron, with a smaller relative relevance of sharp edges in the scattering radiation, we opt only for the hybrid implementation of the CFIE. As shown in Fig. 10, whereas the hybrid RWG/monopolar-RWG implementation provides similar accuracy as CFIE[R], the analogous hybrid implementation for the EFIE does improve the accuracy for the same $1 \lambda$-tetrahedron [18]. Consistently, as regards the error trend versus $N$ observed in Fig. 14, the hybrid implementation of the CFIE offers similar accuracy as CFIE[R]. In contrast, the analogous hybrid implementations of the EFIE or the MFIE provide clearly better accuracy than their RWG-counterparts. Interestingly, in view of Figs. 10 and 14, the optimized CFIE[R][id] implementation shows slightly better performance than the hybrid RWG/monopolar-RWG CFIE-implementations. This may be due to the less relevant sharp-edge influence in the scattering pattern of the $1 \lambda$-tetrahedron. It is clear, thus, that the advantages for the monopolar-RWG implementations in terms of accuracy become more evident for the EFIE [17][18] and for the MFIE [9],[27] than for the CFIE. In any case, a clear improvement is still manifest for the monopolar-RWG implementations of the CFIE, especially in its hybrid form, in the scattering analysis of conductors with corners and sharp edges with subwavelength dimensions. We attribute such performance to the enhancement of the null-field condition at the boundary through the volumetric testing inside the object near sharp edges, where the low-order approximations of the current provide less accuracy [17]. The competitive accuracy exhibited in Figs. 7-14 by the hybrid RWG/monopolar-RWG CFIE-implementations in the analysis of conformal triangulations paves the way for the accurate analysis of non-conformal triangulations arising from the partitioning of complex objects into independently meshed domains (see section 5.5). Note that the standard CFIE[R] and the optimized CFIE[R][id] implementations cannot handle such meshes. 
ScienceDirect (C) : Article published in Journal of Computational Physics, vol. 407, 2020

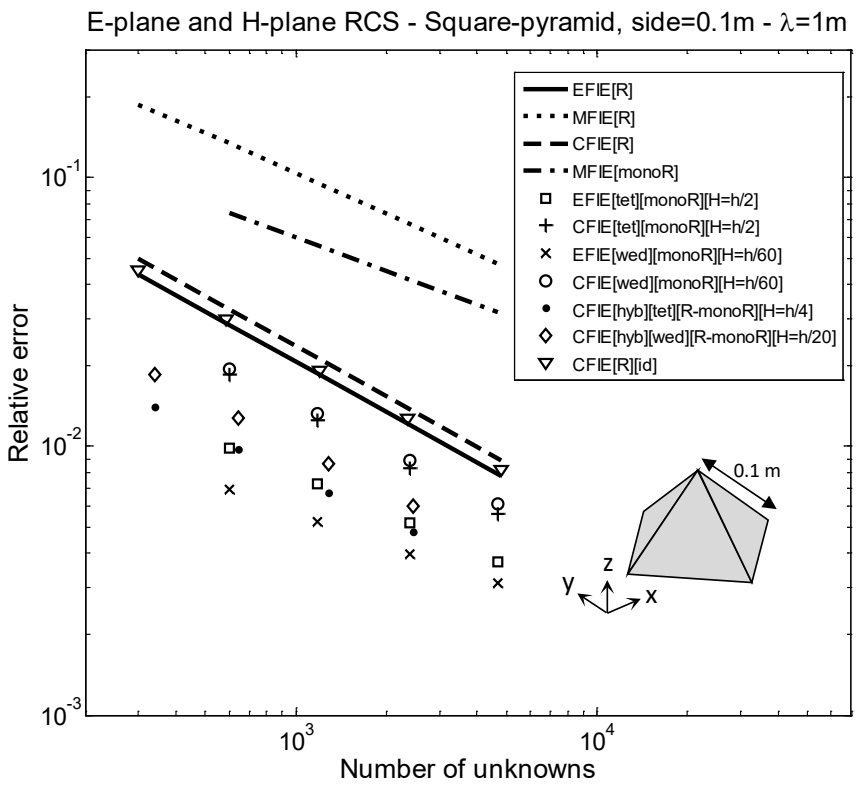

Fig. 12 - Relative RCS-error of several CFIE, EFIE or MFIE implementations, RWG, monopolar-RWG or hybrid RWG/monopolar-RWG, versus the number of unknowns for a square pyramid with side $0.1 \mathrm{~m}(\lambda=1 \mathrm{~m})$. Reference results computed with EFIE[R] and 66918 unknowns.

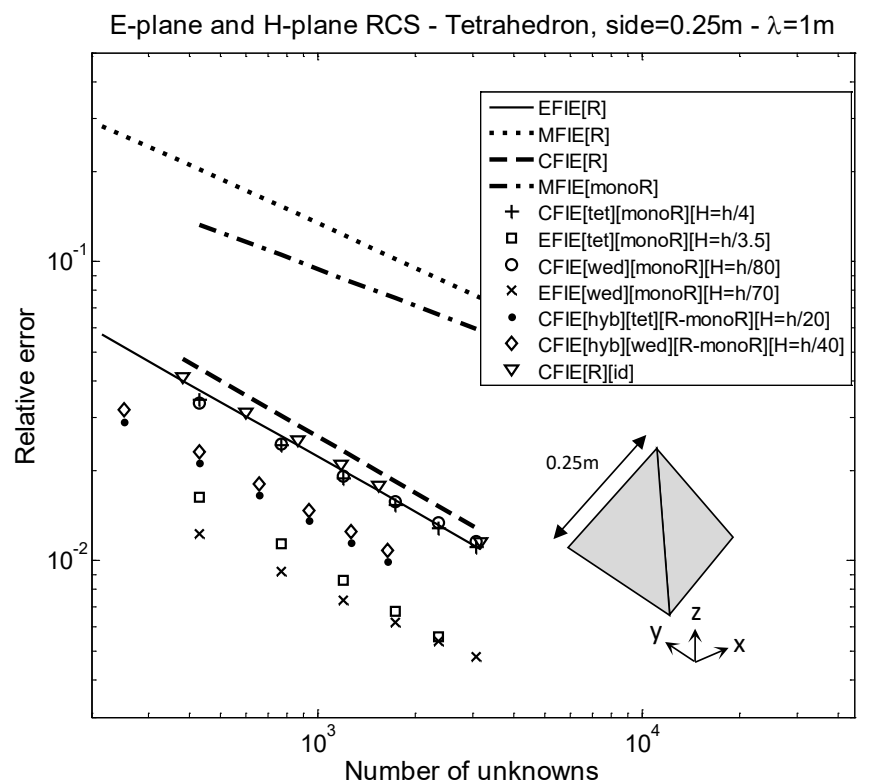

Fig. 13 - Relative RCS-error of several CFIE, EFIE or MFIE implementations, RWG, monopolar-RWG or hybrid RWG/ monopolar-RWG, versus the number of unknowns for a tetrahedron with side $0.25 \mathrm{~m}(\lambda=1 \mathrm{~m})$. Reference results computed with EFIE[R] and 68475 unknowns. 


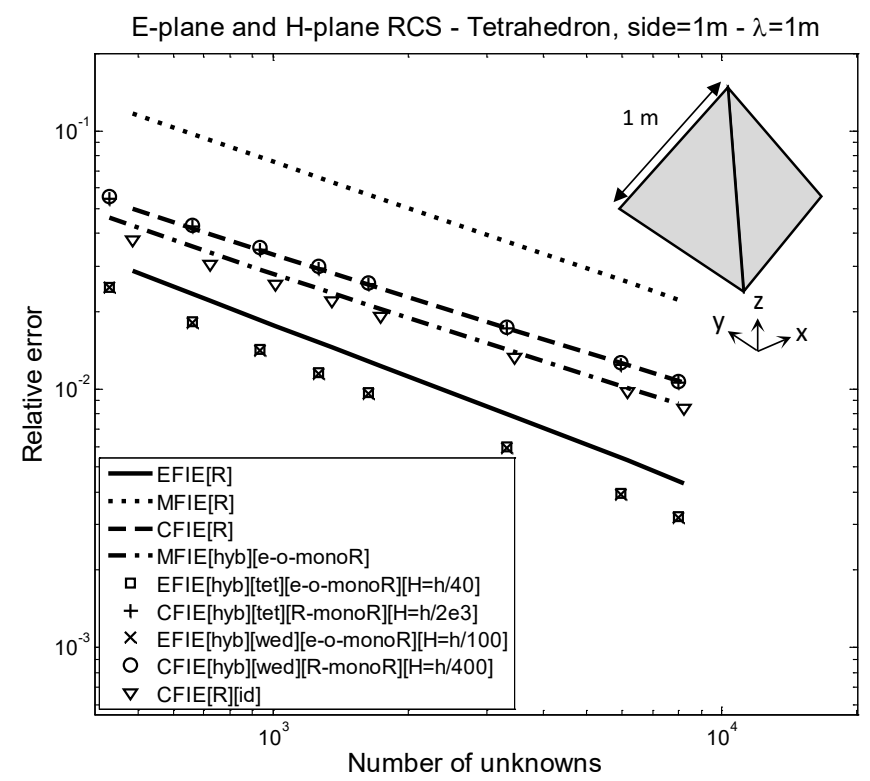

Fig. 14 - Relative RCS-error of several CFIE, EFIE or MFIE implementations, RWG or hybrid RWG/monopolar-RWG, versus the number of unknowns for a tetrahedron with side $1 \mathrm{~m}(\lambda=1 \mathrm{~m})$. Reference results computed with EFIE[R] and 71121 unknowns.

\subsection{Convergence}

We show the number of steps to reach convergence in the iterative search for the solution, under an impinging $\mathrm{x}$ polarized z-propagating wave, for our nonconforming implementations of the CFIE as compared with the conventional RWG-discretization of the EFIE and the CFIE. We perform the iterative search through the generalized minimal residual method [47] and no-restart. The iterative search is not preconditioned and terminates for a residual error below $10^{-4}$. In Figs. 15,16 and 18,19, we display for subwavelength sharp-edged conductors the convergence trends, respectively, versus $H$, and similar number of unknowns, and versus $N$, with the most accurate $H$-values. In light of Figs. 15, 16, the $H$-choice is more critical for the fully monopolar-RWG implementations than for the hybrid RWG-monopolar-RWG implementations. For the $1 \lambda$-tetrahedron, we show in Fig. 17 the number of iterative steps versus $H$ and number of unknowns for the hybrid RWG-monopolar-RWG implementations of the CFIE and the standard RWG-discretization.

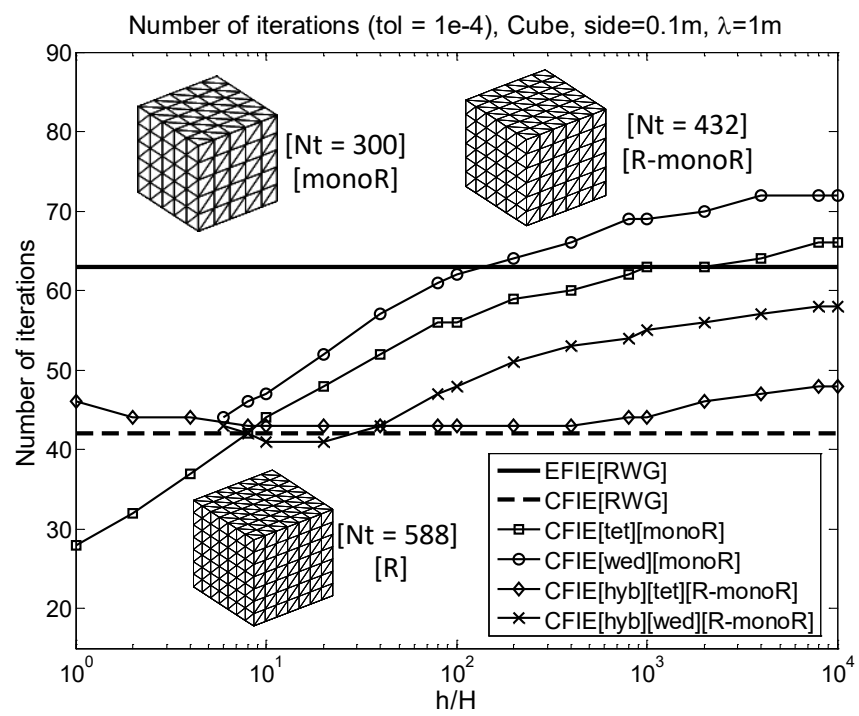

Fig. 15 - Number of steps to reach a residual error below 1e-4 in the GMRES iterative search of the solution for several implementations of the CFIE, monopolar-RWG or hybrid RWG/ monopolar-RWG, with respect to CFIE[R] and EFIE[R] and similar number of unknowns, versus the height $H$ of the testing elements for a cube with side $0.1 \mathrm{~m}(\lambda=1 \mathrm{~m})$ 


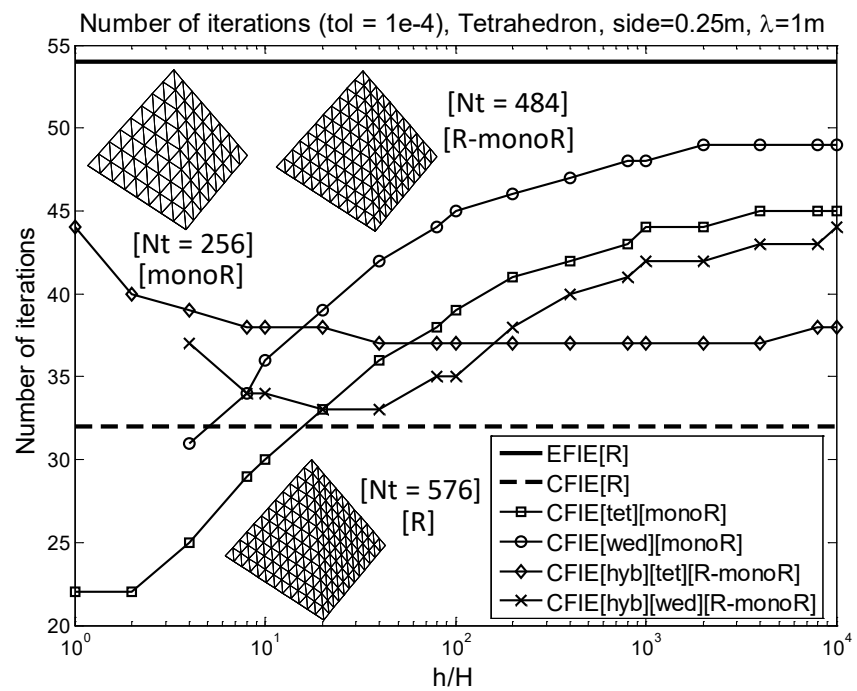

Fig. 16 - Number of steps to reach a residual error below 1e-4 in the GMRES iterative search of the solution for several implementations of the CFIE, monopolar-RWG or hybrid RWG/ monopolar-RWG, with respect to CFIE[R] and EFIE[R] and similar number of unknowns and versus the height $H$ of the testing elements for a tetrahedron with side $0.25 \mathrm{~m}(\lambda=1 \mathrm{~m})$

As is well known, the numerical implementations of the CFIE lead to better-conditioned systems than analogous implementations of the EFIE. Accordingly, the RWG-discretization of the EFIE appears in Figs. 15-19 as the scheme that requires most iterations to converge. In the scattering analysis of subwavelength targets such as the $0.25 \lambda$ tetrahedron and the $0.1 \lambda$-cube, in an analogous manner as with the assessment of the accuracy, the fully monopolarRWG implementations of the CFIE show a varying speed of convergence in terms of the $H$-choice. In light of Figs. 15 and 16, the monopolar-RWG discretization of the CFIE with tetrahedral testing offers better convergence than the conventional RWG-discretization of the CFIE for some specific $H$-ranges that moreover match the $H$-ranges of improved accuracy (see Figs. 7 and 9). In contrast, the monopolar-RWG CFIE-implementation with wedge testing do not show faster convergence than the RWG-discretization of the CFIE or the monopolar-RWG CFIEimplementation with tetrahedral testing. For these electrically small targets, the hybrid RWG-monopolar-RWG CFIE-implementations show, as compared to the monopolar-RWG schemes, a more stable convergence trend in terms of $H$, especially for the hybrid implementation with tetrahedral testing.

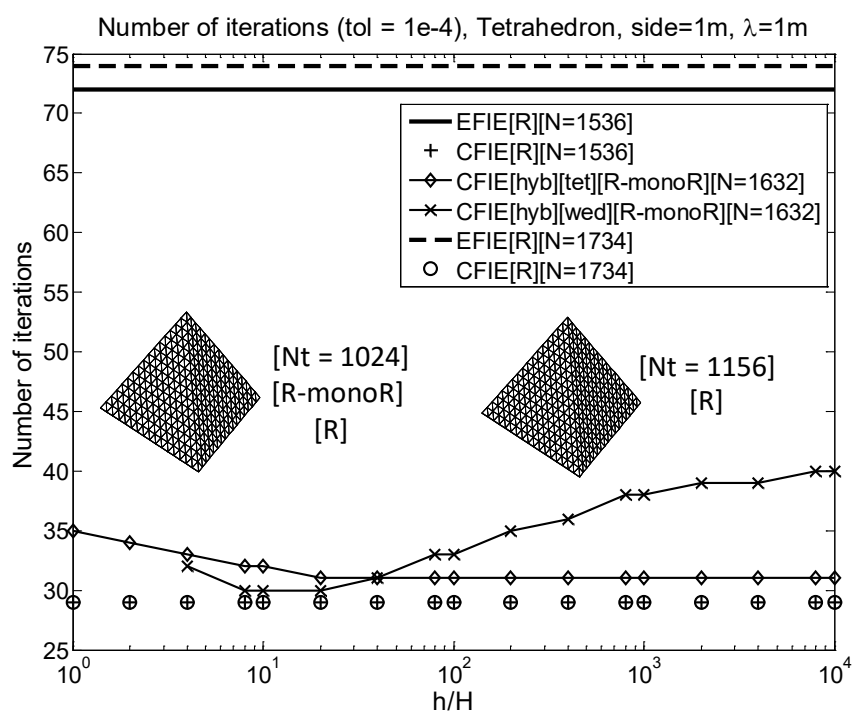

Fig. 17 - Number of steps to reach a residual error below 1e-4 in the GMRES iterative search of the solution for hybrid RWG/monopolar- 
RWG implementations of the CFIE, with respect to CFIE[R] and EFIE[R] and similar number of unknowns $N$, versus the height $H$ of the testing elements for a regular tetrahedron with side $1 \mathrm{~m}(\lambda=1 \mathrm{~m})$

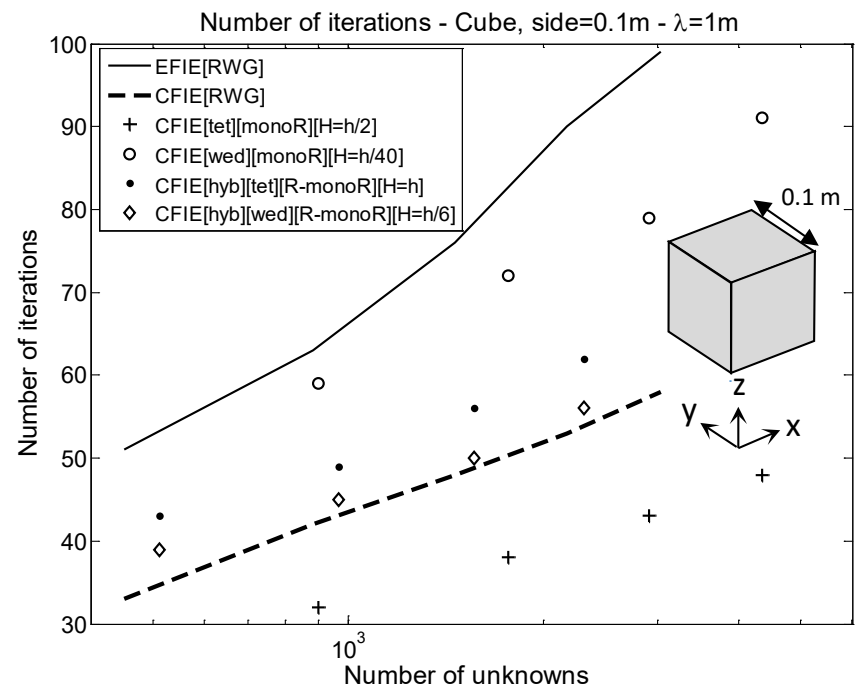

Fig. 18 - Number of iterative steps to reach a residual error below 1e-4 in the iterative search of the solution for several CFIE implementations, monopolar-RWG or hybrid RWG/ monopolar-RWG, versus the number of unknowns for a cube with side $0.1 \mathrm{~m}$ $(\lambda=1 \mathrm{~m})$.

In Figs. 18 and 19, we display for the $0.1 \lambda$-cube and for the $0.25 \lambda$-tetrahedron the convergence trends for the monopolar-RWG CFIE-implementations with respect to the number of unknowns. The monopolar-RWG implementations in Figs. 18 and 19 adopt $H$-values that produce best accuracy. Interestingly, only the tetrahedral choice can provide at the same time improved convergence and accuracy for a particular $H$-value.

For the $1 \lambda$-tetrahedron, as shown in Fig. 17, our hybrid CFIE-implementations offer similar rate of convergence, although slightly slower, than the RWG-discretization of the CFIE. In addition, the convergence trend appears particularly stable in terms of $H$, especially for the tetrahedral scheme of testing, as it happens in an analogous manner with the accuracy (see Fig. 8).

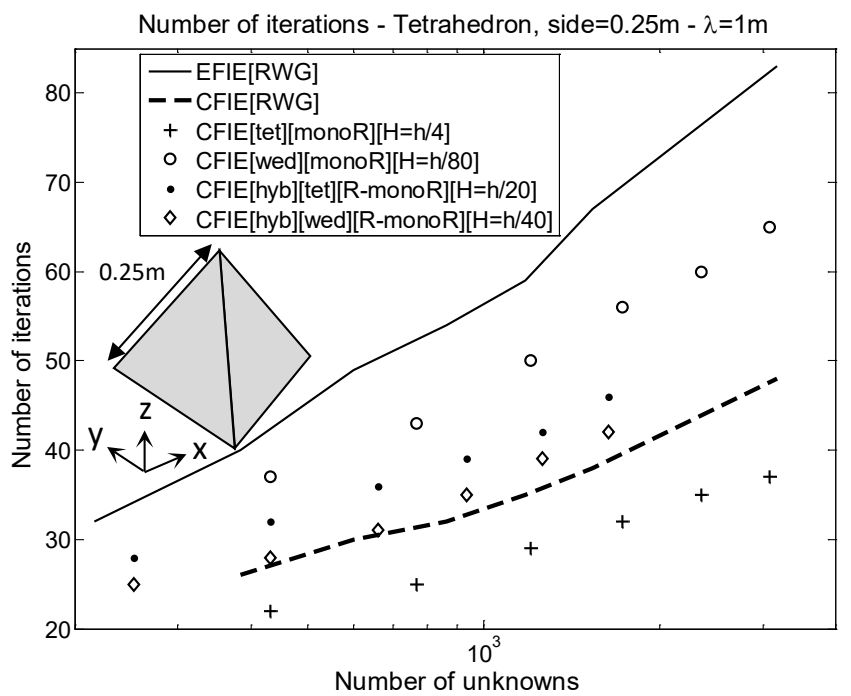

Fig. 19 - Number of iterative steps to reach a residual error below 1e-4 in the iterative search of the solution for several CFIE implementations, monopolar-RWG or hybrid RWG/monopolar-RWG, versus the number of unknowns for a tetrahedron with side $0.25 \mathrm{~m}(\lambda=1 \mathrm{~m})$. 


\subsection{Grid-robustness}

Radio-frequency engineers may encounter defective meshes in the numerical analysis of microwave devices or antennas. A common unambiguous manner to describe a triangular mesh is the connectivity file [48], which comprises two lists: (a) the set of vertices arising in the triangulation and (b) the list of three vertices building each facet. Whereas the connectivity file alone is enough for the facet-based schemes to compute the electromagnetic response, the edge-based schemes require, prior to the scattering analysis, the execution of edge finding algorithms. Conventional IE-solvers require the adopted meshes to be conformal but flaws in the mesh generation (e.g. unconnected vertices or misaligned edges) lead to defective meshes (strictly speaking; non-conformal). The edge finding procedures then provide an incomplete list of interior edges, thereby discarding degrees of freedom in the modelling of the current. In our experience, the evidence in the computed bistatic RCS of this inaccurate modelling of the current depends on several aspects, such as the number of affected mesh elements, the location of the deficiency (over co-planar or sharp-edged facets) or the polarization of the impinging plane wave. On the other hand, flaws in the mesh generation may even become unnoticed by the engineer, especially in the analysis of complex noncanonical structures, with no analytical solutions available. An obvious solution to prevent this is of course to repair the mesh. However, the process of finding and removing ill-shaped triangles, welding disconnected vertices or stitching misaligned edges is normally strenuous and time-consuming, especially, as it often happens, if the mesh has been generated by a third party.

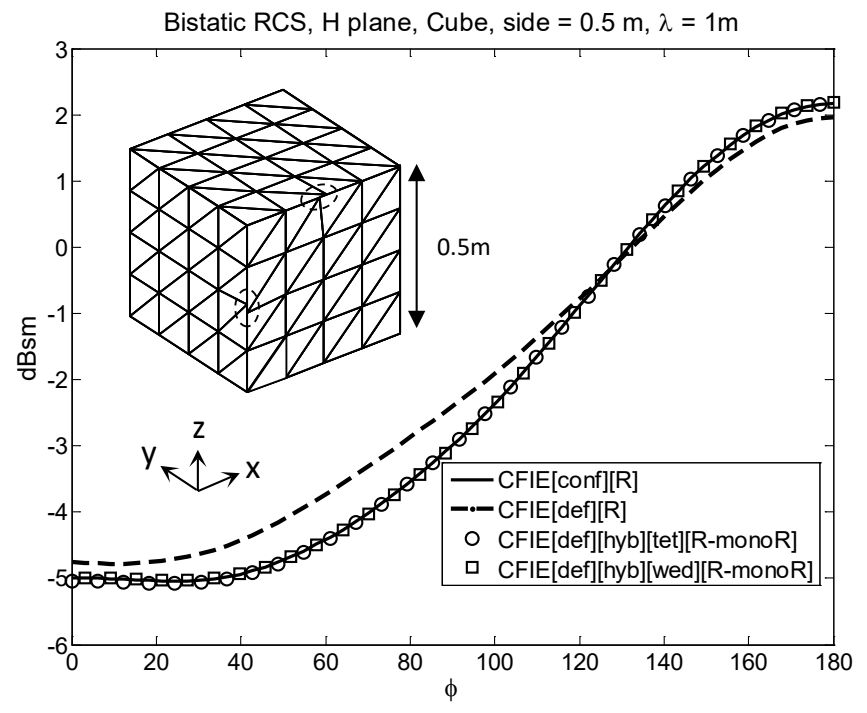

(a)

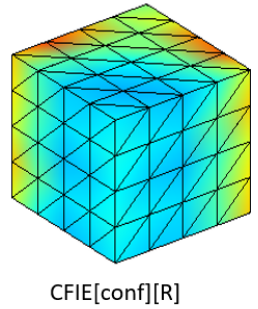

CFIE[def][hyb][tet][R-monoR]

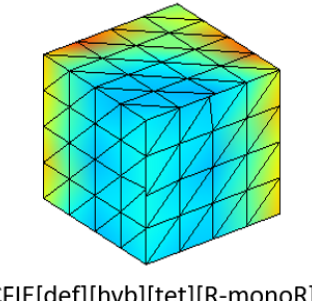

(b)

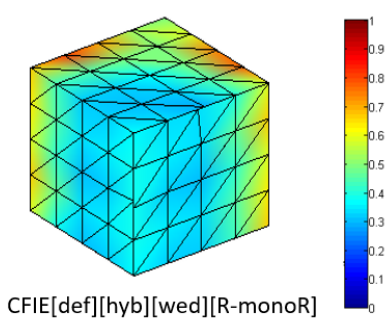

Fig. 20 - (a) - Bistatic RCS over the E plane and (b) Normalized absolute value of the electric current for hybrid RWG/monopolar-RWG CFIE implementations and $H=h / 10$ for a cube with side $0.5 \mathrm{~m}(\lambda=1 \mathrm{~m})$ meshed with a non-conformal defective mesh with two pairs of unconnected vertices The vertices of each pair are shifted $0.0125 \mathrm{~m}$ over opposite directions along the sharp edge. The results are compared with the standard RWG discretization of the CFIE on the original conformal mesh

In Figs. 20 and 21, we display (a) the computed RCS and (b) the electric current distribution for a cube meshed with 192 triangles after distorting the original mesh on two vertices located on sharp edges or corners. In Fig. 20 a 
couple of vertices located in the middle of two sharp edges are split into pairs of unconnected vertices slightly shifted along the sharp edge, giving rise to so-called T-junctions [6] . In Fig. 21 tiny slits are inserted at two corners of the original mesh. The latter represents a more harmful modification because of the outbreak of spurious slits that convert the original closed geometry into an unwanted open surface. For this reason, the adopted dimensions for the slits in Fig. 21 are much smaller than the shift of the unconnected vertices in Fig. 20. In view of Figs. 20-(a) and 21(a), the RCS computed with the edge-based CFIE[R] for both defective meshes results in remarkable inaccuracies when compared with the original conformal mesh. We activate these inaccuracies with an appropriate plane wave choice $(\hat{e}=(-\hat{x}+\hat{y}) / \sqrt{2} ; \hat{h}=-\hat{z} ; \hat{k}=-(\hat{x}+\hat{y}) / \sqrt{2})$. The erroneous analysis with CFIE[R] of the defective meshes in Figs. 20(a) and 21-(a) results from the misclassification as boundary edges of a few interior edges. Furthermore, the CFIE[R] analysis of the mesh in Fig. 21 is specifically sensitive to the spurious electromagnetic scattering effect of the slits. In view of Figs. 20 and 21, our hybrid monopolar-RWG schemes appear immune to the existence of such deficiencies in the mesh. This represents progress with respect to previous patch-based IE-schemes reporting gridrobustness only for smooth conductors [10].

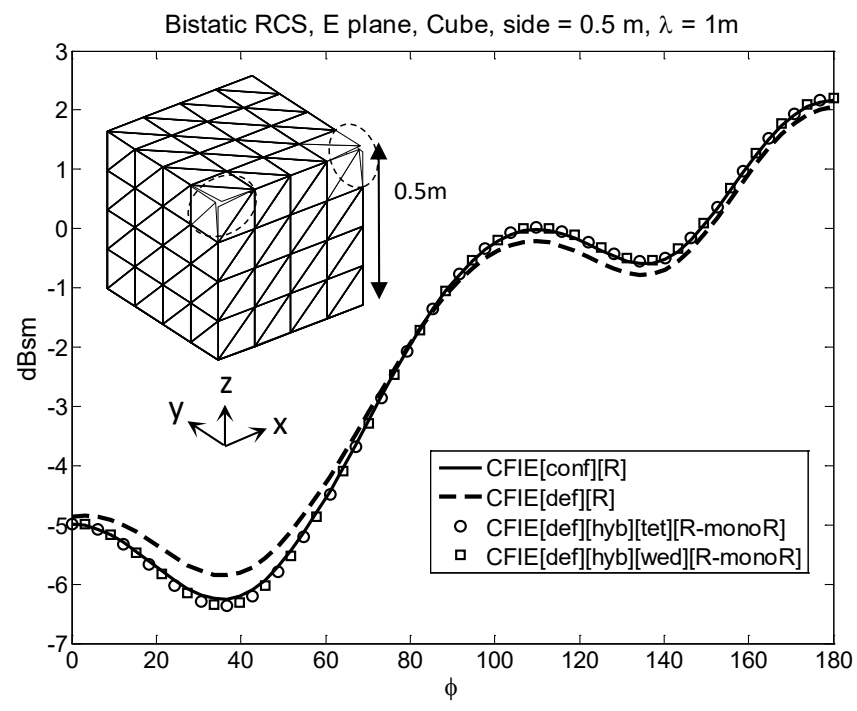

(a)

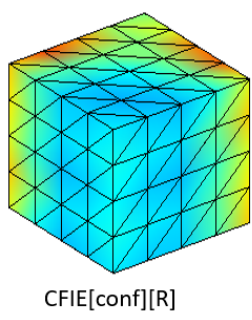

CFIE[def][hyb][tet][R-monoR]

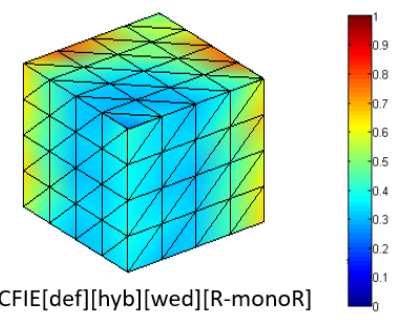

(b)

Fig. 21 - (a) Bistatic RCS over the E plane and (b) Normalized absolute value of the electric current for hybrid RWG/monopolar-RWG CFIE implementations and $H=h / 10$ for a cube with side $0.5 \mathrm{~m}(\lambda=1 \mathrm{~m})$ meshed with a non-conformal defective mesh where 0.00125 -meter long slits are inserted at two corner vertices. The results are compared with the standard RWG discretization of the CFIE on the original conformal mesh

\subsection{Versatility}

Our facet-based monopolar-RWG CFIE-implementations allow the analysis of meshed structures with non-nonconformal transitions that the conventional edged-based schemes cannot consider. Moreover, unlike other facetbased schemes [37], our schemes do not need to impose additional conditions across domain boundaries. In Figs. 22-26, we show (a) RCS and (b) electric current results, under an impinging x-polarized z-propagating wave, for our hybrid CFIE-implementations (and $H=h / 10$ ) for meshes that originate from the juxtaposition of triangulations 
associated with the domains that take part in the decomposition of the objects. These meshes are by construction non-conformal across the boundaries between touching domains. We then define monopolar-RWG basis functions (and the volumetric testing) on triangle edges matching the domain boundaries and RWG basis functions (and Galerkin testing) elsewhere. We validate the RCS-results computed with our hybrid CFIE-implementations against RWG-results, which require conformal meshes.

In Figs. 22 and 23, the objects under analysis, a square pyramid (with no basis) on top of a prism (with no top face) and a tetrahedron, give rise to meshes where the facets touching the boundaries have edges fully matching the boundary. In Figs 24-25, though, the tested objects are curved and the facets at both sides of the boundaries do not fully match, whereby the outbreak of spurious slits is inevitable. The analysis of the non-conformal meshes for the objects with no slits in Figs. 22-23 show very similar performance as the conventional analysis on conformal meshes. In our experience, the performance for our hybrid CFIE-implementations for non-conformal meshes with spurious slits, appears acceptable but somewhat less accurate. Interestingly, the results appear a bit more reliable for the cone-cylinder in Fig. 24 - i.e. a cone (with no basis) on top of a cylinder (with no top face and same radius) than for the sphere in Fig. 25. In general, as is well known, the use of non-conformal triangulations for modelling curved shapes, especially with small radius of curvature, although quite often implemented in practice, is not the best choice because of the advent of spurious sharp mesh edges.

In Fig. 26, we show results for a PEC torus, an example of non-simply connected structure, with major radius $\lambda / 3$ and minor radius $\lambda / 6(\lambda=1 \mathrm{~m})$ [49]. The hybrid-CFIE analysis of the non-conformal mesh arising from assigning different triangulations to each half of the torus provides similar accuracy as the conventional analysis with a conformal mesh. Even though this torus has moderately small dimensions, the computed results are free from the inaccuracies reported for the MFIE in the static limit analysis [50]. In any case, this frequency region is out of the performance band of conventional CFIE-implementations as full-wave solvers.

In Fig. 27, we present RCS results for a large PEC cone-sphere, with diameter $3 \lambda$ and angle $75^{\circ}(\lambda=1 \mathrm{~m})$. The cone-sphere is a shape formed by joining a cone and a sphere so that the radius and its first derivative are matched at the cone-sphere transition [51]. This is a quite a demanding case, as far as accuracy is concerned, because the backscattered RCS is low, roughly $40 \mathrm{~dB}$ below the forward-scattered RCS. Our hybrid RWG/monopolar-RWG implementations makes use of a non-conformal mesh (with a non-conformal transition between the sphere and cone regions) and the RWG-discretization of the CFIE manages a conformal mesh (see Fig. 28). Whereas the nonconformal mesh employs 27774 triangular facets, leading to a matrix system of 38799 unknowns, the conformal mesh employs 35904 unknowns, with a resulting matrix system of 53856 unknowns, at the limit of our available memory resources to compute the direct solution. Our conformal-mesh generating scheme gives rise to a very fine mesh over the cone-region that we relax in the non-conformal mesh generation by assigning somewhat looser mesh criteria to the sphere- and cone-regions (see Fig. 28). The mesh relaxation criterion is in any case very subtle; the maximum mesh edge lengths amount to $0.1100 \lambda$ for the conformal mesh and to $0.1157 \lambda$ for the non-conformal mesh. In view of Fig. 27, there is a significant similarity between the results computed with the hybrid RWG/monopolarRWG CFIE-discretization and the standard RWG-discretization. Note that the match in Fig. 27 is much tighter in relative terms than in Fig. 25, for the electrically small sphere. We attribute this to the fact that the sphere in Fig. 25 has a diameter of $3 \lambda$ and therefore an electrically bigger radius of curvature than the sphere in Fig. 25, with a diameter of $0.4 \lambda$, which suffers from a somewhat rougher flat-facetted modelling of the boundary surface. 
ScienceDirect (C) : Article published in Journal of Computational Physics, vol. 407, 2020

https://doi.org/10.1016/j.jcp.2020.109236

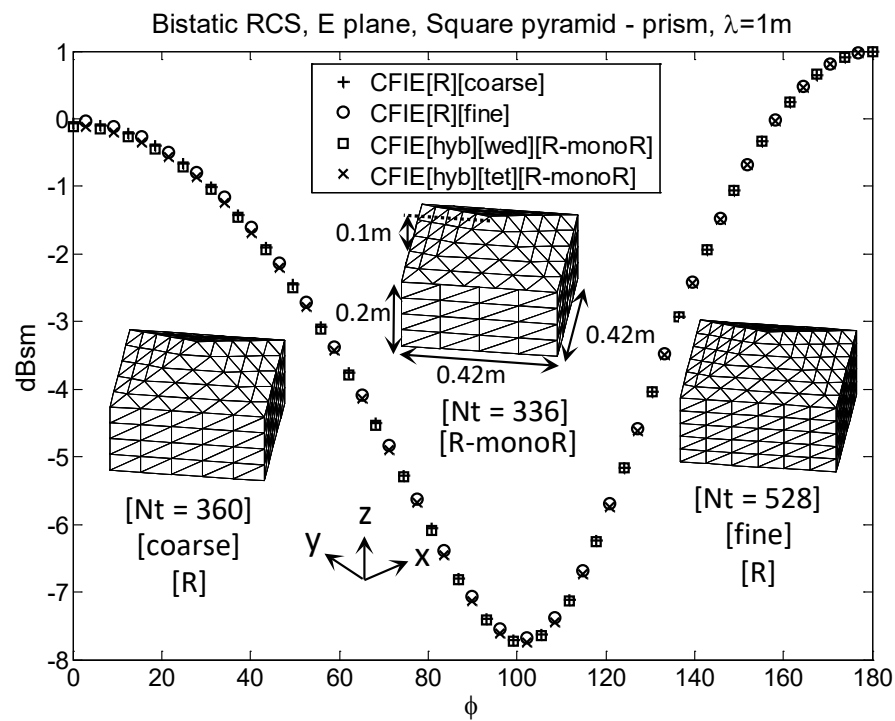

(a)

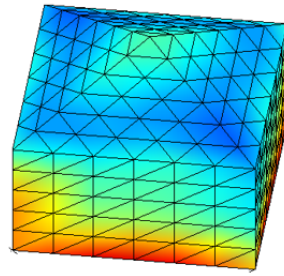

CFIE[R][fine]

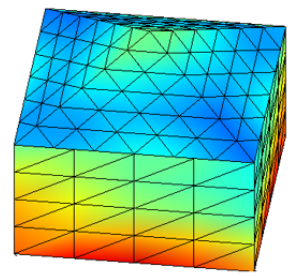

CFIE[hyb][tet][R-monoR]

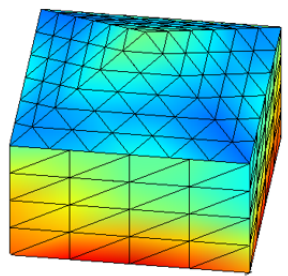

CFIE[hyb][wed][R-monoR]

(b)

Fig. 22 - (a) Bistatic RCS over the E plane and and (b) Normalized absolute value of the electric current for hybrid RWG/monopolar-RWG CFIE implementations and $H=h / 10$ for a composite object arising from the juxtaposition of a square pyramid on top of a prism $(\lambda=1 \mathrm{~m})$ and a non-conformal mesh. The results are compared with the standard RWG discretization of the CFIE for two conformal meshes. The impinging plane wave is z-propagating and x-polarized.

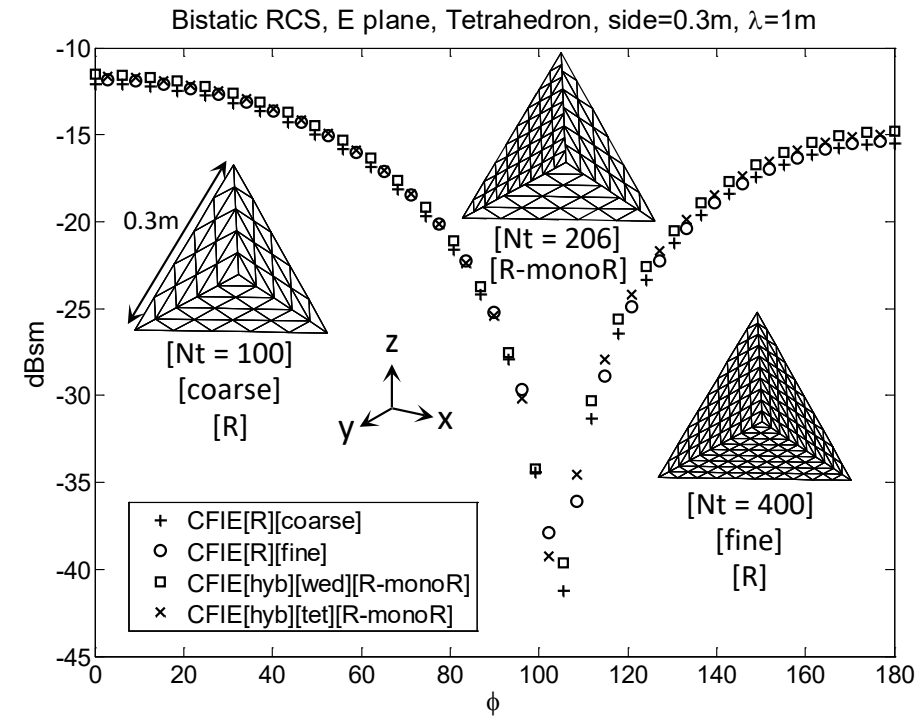

(a) 


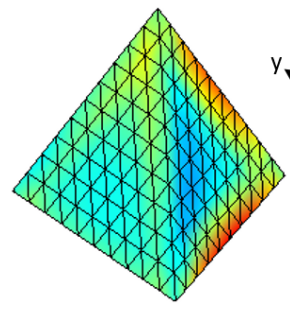

CFIE[R][fine]

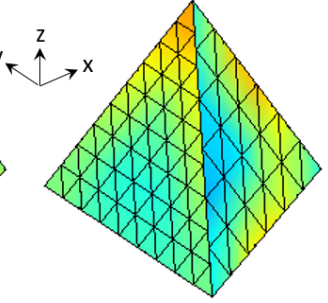

CFIE[hyb][tet][R-monoR]

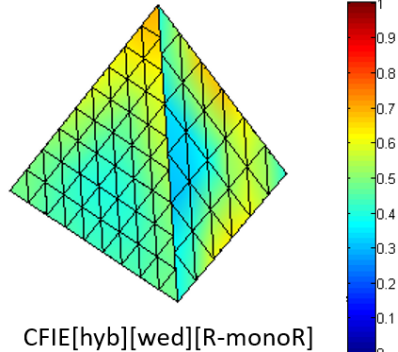

(b)

Fig. 23 - (a) Bistatic RCS over the E plane and (b) Normalized absolute value of the electric current for hybrid RWG/monopolar-RWG CFIE implementations and $H=h / 10$ for a tetrahedron with side $0.3 \mathrm{~m}(\lambda=1 \mathrm{~m})$ meshed through a non-conformal transition over the sharp edges. The results are compared with the standard RWG discretization of the CFIE for two conformal meshes. The impinging plane wave is z-propagating and x-polarized.

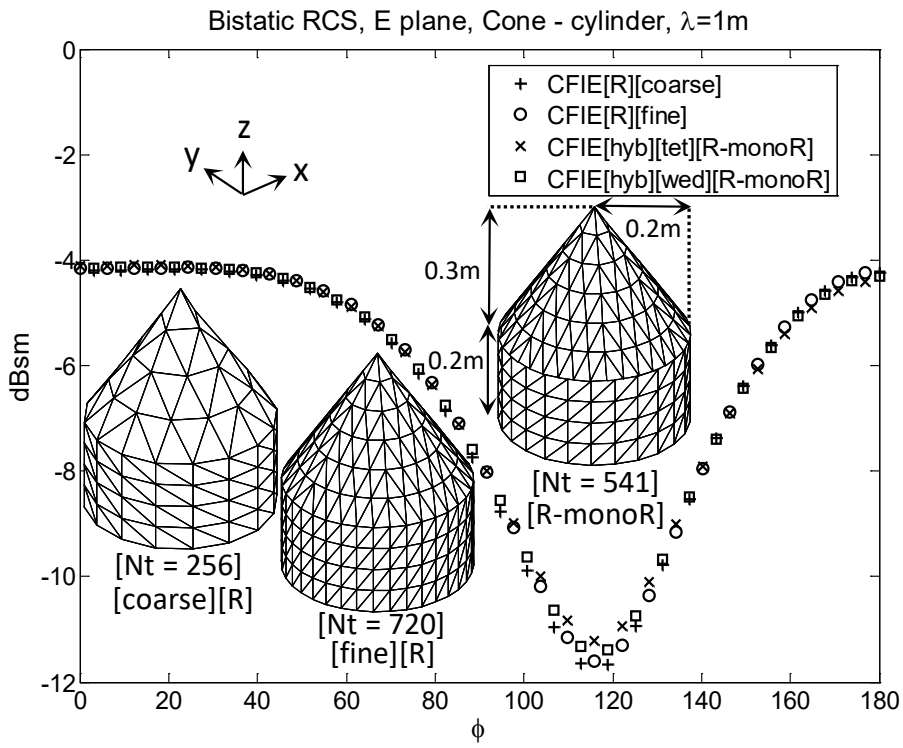

(a)

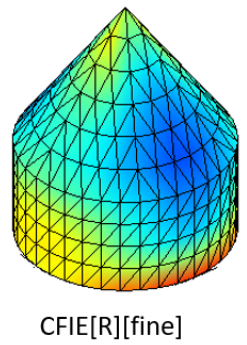

CFIE[R][fine]

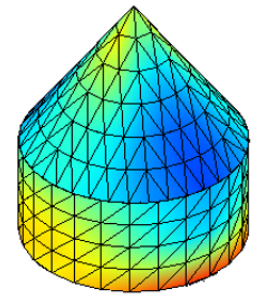

CFIE[hyb][tet][R-monoR]

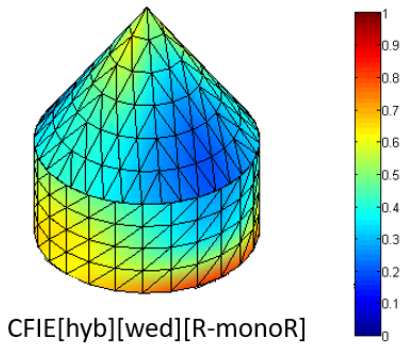

CFIE[hyb][wed][R-monoR]

(b)

Fig. 24 - (a) Bistatic RCS over the E plane and (b) Normalized absolute value of the electric current for hybrid RWG/monopolar-RWG CFIE implementations and $H=h / 10$ for a cone-cylinder meshed through a non-conformal transition over the sharp intersecting boundary line. The results are compared with the standard RWG discretization of the CFIE for two conformal meshes. The impinging plane wave is z-propagating and x-polarized. 
ScienceDirect (C) : Article published in Journal of Computational Physics, vol. 407, 2020

https://doi.org/10.1016/j.jcp.2020.109236

Bistatic RCS, E plane, Sphere, radius $=0.2 \mathrm{~m}, \lambda=1 \mathrm{~m}$

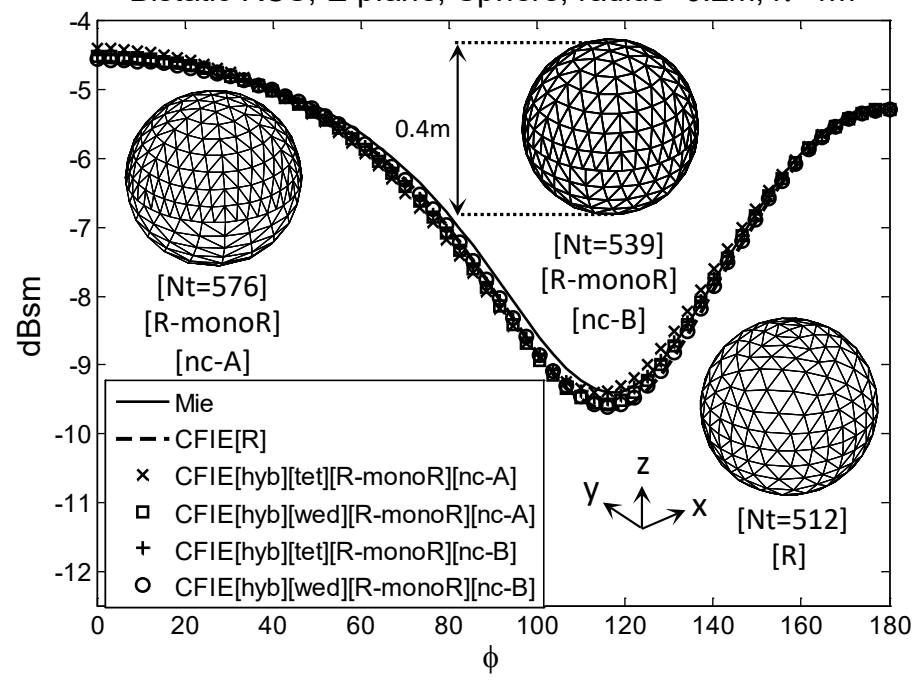

(a)

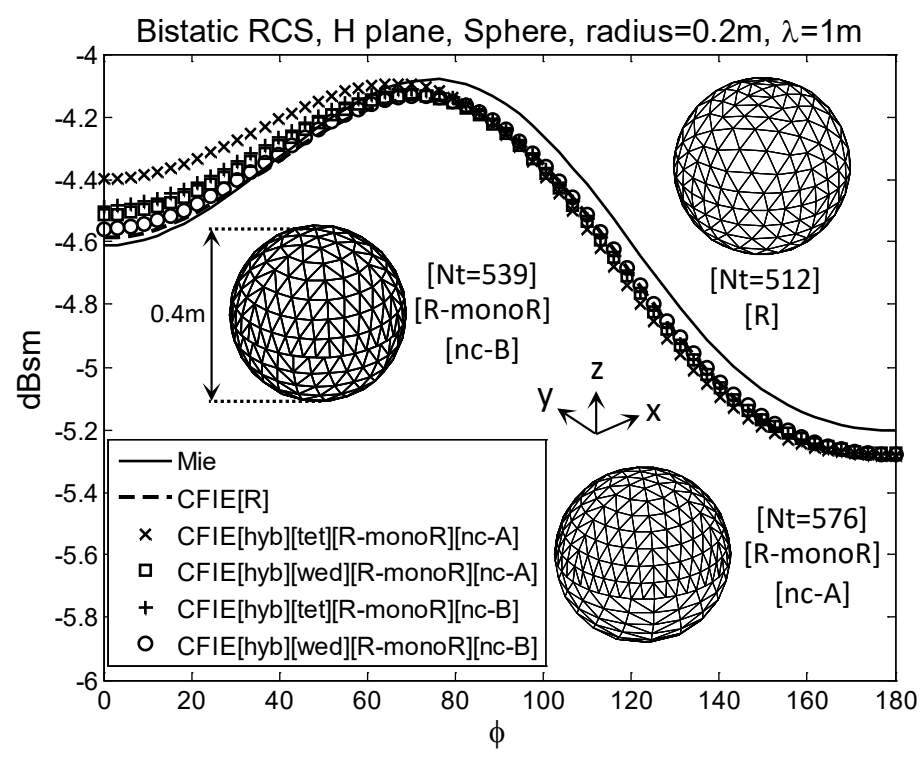

(b)

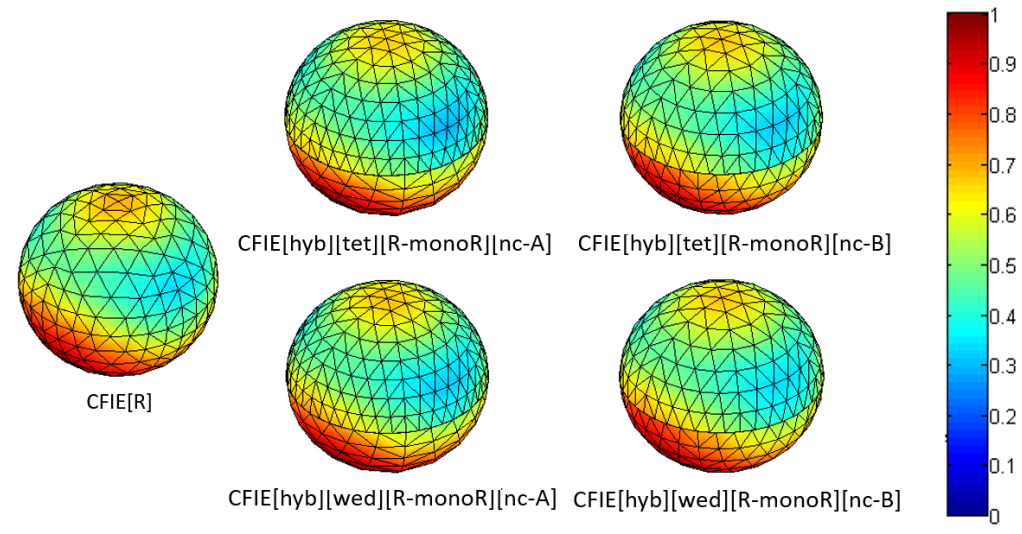

(c) 
Fig. 25 - (a) RCS over the E plane, (b) RCS over the H plane and (c) Normalized absolute value of the electric current for hybrid RWG/monopolar-RWG CFIE implementations and $H=h / 10$ for a sphere with radius $0.2 \mathrm{~m}$ and two non-conformal transitions between the two hemispheres. The results are compared with the RWG discretization of the CFIE on a conformal mesh and the Mie series solution. The impinging plane wave is z-propagating and x-polarized.

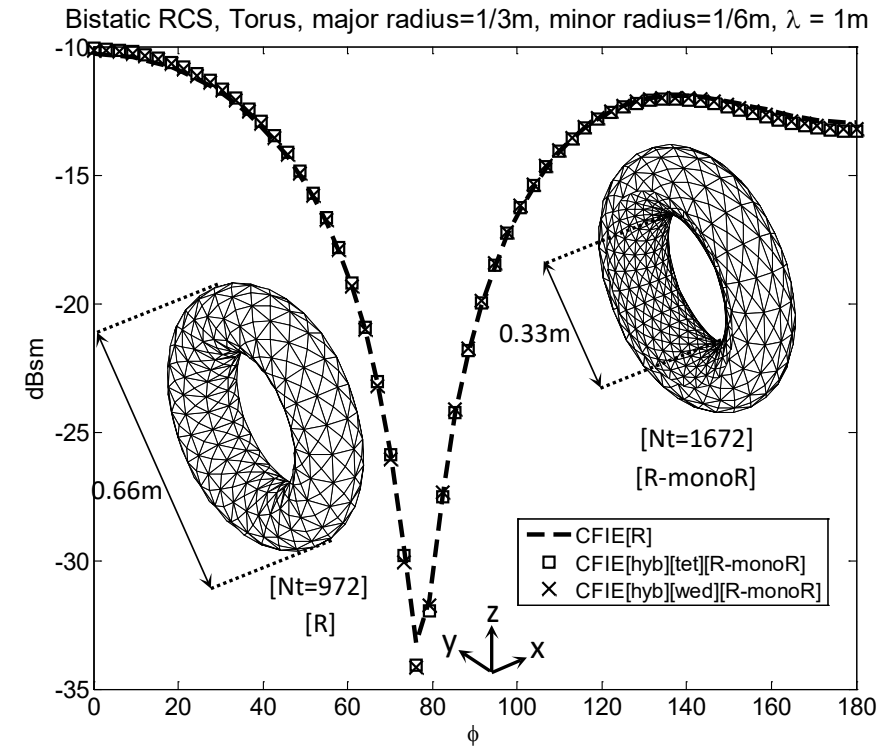

(a)
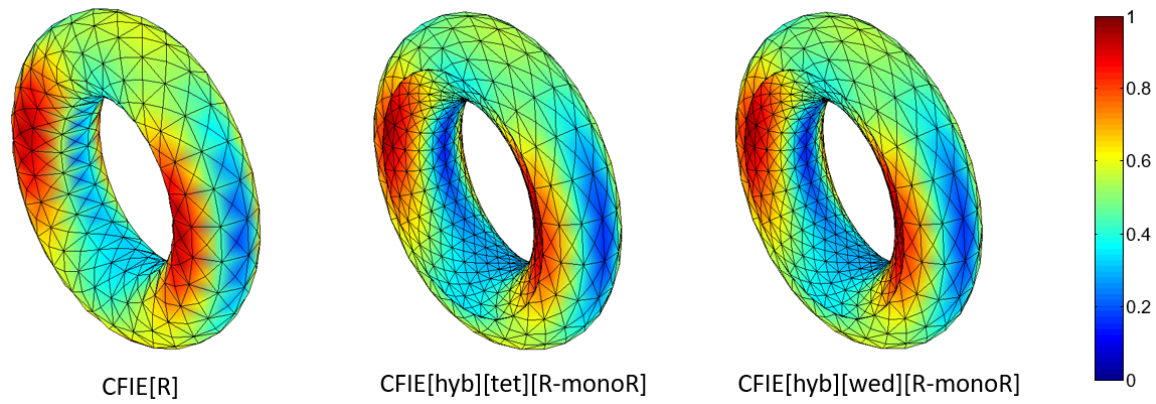

(b)

Fig. 26 - (a) RCS over the H plane and (b) Normalized absolute value of the electric current for hybrid RWG/monopolar-RWG CFIE implementations and $H=h / 10$ for a torus with major radius $\lambda / 3$ and minor radius $\lambda / 6$ meshed with non-conformal transition between two halves. The results are compared with the RWG discretization of the CFIE on a conformal mesh. The impinging plane wave is z-propagating and x-polarized. 
ScienceDirect (C) : Article published in Journal of Computational Physics, vol. 407, 2020

Bistatic RCS, E plane, Cone-sphere, radius $=1.5 \mathrm{~m}$, angle $=75^{\circ}$

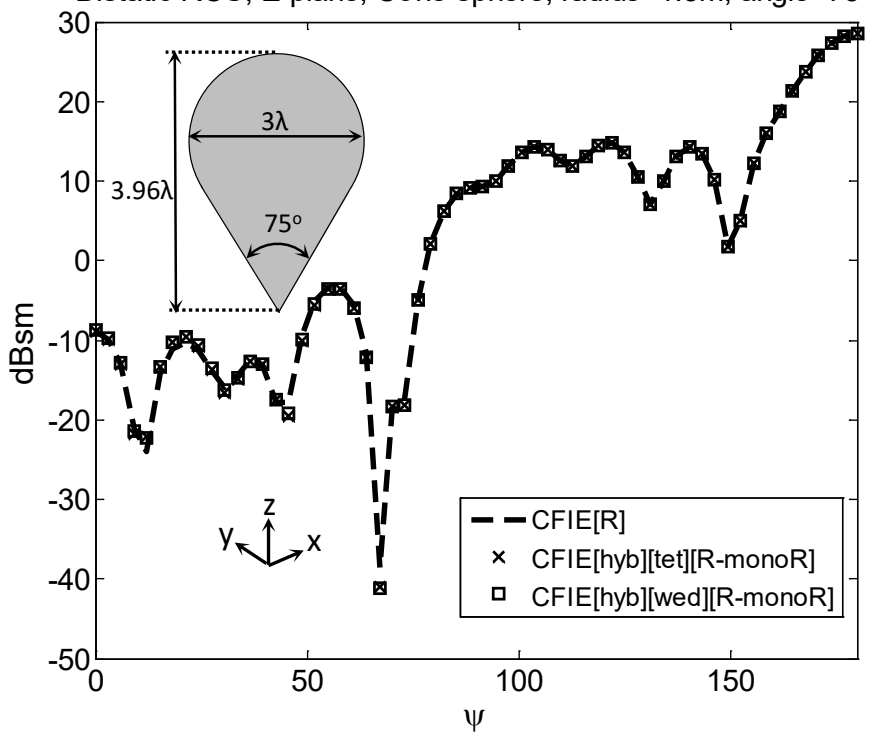

Fig. 27 - (a) RCS over the E plane and (b) Normalized absolute value of the electric current for hybrid RWG/monopolar-RWG CFIE implementations and $H=h / 10$ for a cone-sphere with diameter $3 \lambda$ and angle $75^{\circ}$ meshed with non-conformal transition between the sphere and the cone parts $(\mathrm{Nt}=27774)$. The results are compared with the RWG discretization of the CFIE on a conformal mesh $(\mathrm{Nt}=35904)$. The impinging plane wave is z-propagating and x-polarized $(\lambda=1 \mathrm{~m})$.

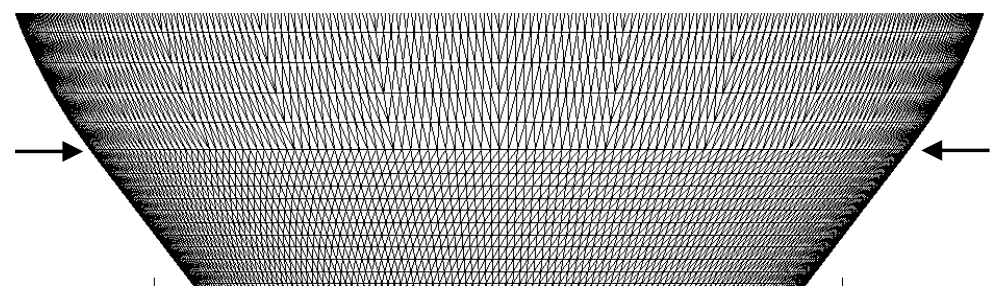

(a)

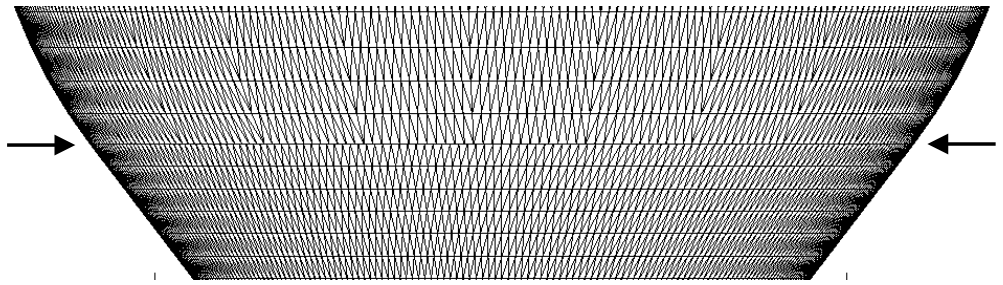

(b)

Fig. 28 - Mesh transitions for the triangulations adopted for the scattering analysis of a cone-sphere with diameter $3 \lambda$ and angle $75^{\circ}$ : (a) Conformal ( $\mathrm{Nt}=35904)$; (b) Non-conformal ( $\mathrm{Nt}=27774)$. 


\section{Conclusion}

We present a new discretization of the CFIE based on the facet-based monopolar-RWG basis functions, discontinuous across edges. Our implementation arises from the combination of volumetrically tested electric-field contributions and Galerkin tested magnetic-field terms. The volumetric subdomains of electric-field testing, tetrahedral elements or wedges, are attached to the surface triangulation, inside the conductor. For subwavelength sharp-edged conductors, our monopolar-RWG CFIE-implementations exhibit better accuracy than the standard edgebased RWG-discretization of the CFIE for particular ranges of heights $H$ of the testing subdomains (set in terms of the mesh parameter $h$ ). The tetrahedral and wedge testing schemes offer best improved performance for $H$ ranges, respectively, around $h / 10$ and $h / 100$. These trends become more evident for objects with pronounced sharp edges. Our tests also show that in these cases the observed accuracy becomes even competitive with respect to the RWG discretization of the EFIE. Interestingly, the CFIE-implementation with tetrahedral testing, for the electrically small sharp-edged conductors tested, shows improved speed of convergence in the iterative search of the solution, with respect to the RWG-discretization of the CFIE, for similar $H$-ranges as for the observed improved accuracy.

In a similar manner as observed for the monopolar-RWG implementations of the EFIE or the MFIE, the improved accuracy for our monopolar-RWG implementations of the CFIE becomes decreasingly relevant as the electrical dimensions of the sharp-edged target rise. We attribute this to the reduced relative contribution of the sharp edges in the EM scattering of such objects. Hence, the observed improved accuracy cannot make up for the increase in number of unknowns as the electrical dimensions of the object rise. In such cases, the adoption of hybrid RWG/monopolar-RWG CFIE-implementations appears justified. The current is expanded with the RWG set over edges between co-planar (or almost co-planar) facets and with the monopolar-RWG choice at triangles matching a sharp edge of the target. The number of unknowns of the standard RWG and the hybrid monopolar-RWG discretizations becomes then similar and the observed accuracy remains similar as the fully discontinuous (across edges) approach but with less unknowns. In consequence, the observed $H$ ranges of improved accuracy for subwavelength sharp-edged targets become much broader and the $H$-choice appears less critical. Typically, we adopt $H$ values around $h / 10$ or $h / 100$ for the hybrid RWG/monopolar-RWG discretization of the CFIE with, respectively, tetrahedral or wedge testing. Moreover, for electrically moderate or big sharp-edged objects, the hybrid schemes show similar accuracy and speed of convergence as the RWG discretization of the CFIE. In general, we remark that the relative accuracy improvement with respect to the RWG counterpart, if observed, is smaller for the full or hybrid monopolar-RWG implementations of the CFIE than for the analogous implementations of the EFIE or the MFIE alone.

Our CFIE schemes are facet-based and ignore by definition edges in the establishment of the set of unknowns. They appear then inherently favorable for the analysis of slightly defective meshes that otherwise lead to an incorrect edge identification and perhaps inaccuracies in the analysis with the traditional edge-based tools. Furthermore, our schemes offer enhanced versatility, as compared with the traditional edge-based schemes, in the management of complex geometrically conformal or non-conformal meshes. Our schemes can undertake with good accuracy the modular analysis of complex objects through the partitioning into independently meshed domains without introducing additional unknowns or continuity conditions along the intersecting boundary lines. The hybrid RWG/monopolar-RWG schemes appear especially well suited by defining the RWG discretization over pairs of triangles inside the partitioning domains and by associating the monopolar-RWG discretization strictly with the facet edges matching the non-conformal mesh transitions between adjacent domains.

\section{Acknowledgment}

This work was supported by FEDER and the Spanish Comisión Interministerial de Ciencia y Tecnología (CICYT) under projects TEC2016-78028-C3-1-P , TEC2017-84817-C2-2-R and the Unidad de Excelencia Maria de Maeztu MDM-2016-0600, funded by the Agencia Estatal de Investigación, Spain. 


\section{References}

[1] A.J. Poggio, E.K. Miller, Integral equation solutions of three- dimensional scattering problems, in: R. Mittra (Ed.), Computer Techniques for Electromagnetics, Pergamon Press, Oxford, 1973 (Ch. 4).

[2] Andrew F. Peterson, "The "interior resonance" problem associated with surface integral equations of electromagnetics: Numerical consequences and a survey of remedies", Electromagnetics, vol. 10, No. 3, pp. 293-312, 1990.

[3] J. R. Mautz and R. F. Harrington, "H-field, E-field, and combined-field solutions for conducting bodies of revolution," Archiv für Elektronik und Übertragungstechnik (Electronics and Communication), vol. 32, no. 4, pp. 157-164, 1978.

[4] R. D. Graglia, D. R. Wilton and A. F. Peterson, "Higher Order Interpolatory Vector Bases for Computational Electromagnetics", IEEE Transactions on Antennas and Propagation, vol. 45, No. 3, pp. 329-342, March 1997.

[5] S. M. Rao, D. R. Wilton and A. W. Glisson, "Electromagnetic Scattering by surfaces of Arbitrary Shape", IEEE Transactions on Antennas and Propagation, vol. AP-30, No. 3, pp. 409-418, May 1982.

[6] W. C. Gibson, The Method of Moments in Electromagnetics (Chapman and Hall/CRC, 2008)

[7] Mario A Echeverri Bautista, Francesca Vipiana, Matteo Alessandro Francavilla, Jorge A. Tobon Vasquez, and Giuseppe Vecchi,, "A Nonconformal Domain Decomposition Scheme for the Analysis of Multiscale Structures", IEEE Transactions on Antennas and Propagation, Vol.. 63, No. 8, pp. 3548- 3560, August 2015.

[8] P. Ylä-Oijala, M. Taskinen, and J. Sarvas, "Surface integral equation method for general composite metallic and dielectric structures with junctions," Progress In Electromagnetics Research, Vol. 52, pp. 81-108, 2005.

[9] E. Ubeda, J.M. Rius, "Novel monopolar MoM-MFIE discretization for the scattering analysis of small objects", IEEE Transactions on Antennas and Propagation, vol. 54, No. 1, pp. 50.57, January 2006.

[10] Gang Kang, Jiming Song, Weng Cho Chew, Kalyan C. Donepudi, and Jian-Ming Jin, "A novel grid-robust higher order vector basis function for the method of moments," IEEE Trans. Antennas Propag., vol. 49, no. 6, , pp. 908-914, June 2001

[11] I. Sekulic, E. Ubeda, and J. M. Rius, "Versatile and accurate schemes of discretization in the scattering analysis of 2D composite objects with penetrable or perfectly conducting regions," IEEE Transactions on Antennas and Propagation,, vol. 65, No. 5, pp. 2494-2506, May 2017.

[12] I. Sekulic, E. Ubeda, and J. M. Rius, "Versatile and accurate schemes of discretization for the electromagnetic scattering analysis of arbitrarily shaped piecewise homogeneous objects," Journal of Computational Physics 374 (2018) 478-494.

[13] Lawrence F. Canino, John J. Ottusch, Mark A. Stalzer, John L. Visher, and Stephen M. Wandzura, "Numerical Solution of the Helmholtz Equation in 2D and 3D Using a High-Order Nyström Discretization," Journal of Computational Physics 146, 627-663, 1998.

[14] Andrew F. Peterson and Malcolm M. Bibby, An introduction to the Locally-Corrected Nystrom Method, Morgan \& Claypool Publishers, 2010.

[15] Mei Song Tong, Weng Cho Chew, "A novel approach for evaluating hypersingular and strongly singular surface integrals in electromagnetics", IEEE Transactions on Antennas and Propagation, vol. 58, No. 11, pp. 3593-3601, November 2010.

[16] Z. Peng, K.H. Lim, J.F. Lee, "A discontinuous Galerkin surface integral equation method for electromagnetic wave scattering from nonpenetrable targets", IEEE Transactions on Antennas and Propagation vol.6, No. 7, pp. 3617-3628, July 2013.

[17] E. Ubeda, J.M. Rius, A. Heldring, "Nonconforming discretization of the electric-field integral equation for closed perfectly conducting objects, "IEEE Transactions on Antennas and Propagation, vol. 62, No. 8, pp. 4171-4186, August 2014.

[18] E. Ubeda, J.M. Rius, A. Heldring, I. Sekulic, "Volumetric testing parallel to the boundary surface for a nonconforming discretization of the electric-field integral equation", IEEE Transactions on Antennas and Propagation, vol.. 63, No. 7, pp. 3286-3291, July 2015.

[19] E. Ubeda, I. Sekulic, A. Heldring, and J. M. Rius, "Tangential-normal surface testing for the nonconforming discretization of the electricfield integral equation," IEEE Antennas and Wireless Propagation Letters, vol. 15, pp. 1581-1584, 2016.

[20] Gaobiao Xiao, Yibei Hou, "Intuitive formulation of discontinuous Galerkin surface integral equations for electromagnetic scattering problems", IEEE Transactions on Antennas and Propagation, vol. 65, No. 1, pp. 287-294, January 2017.

[21] Eduard Ubeda, Alex Heldring and Juan M. Rius, "Accurate computation of the impedance elements of the magnetic-field integral equation with RWG basis functions through field-domain and source-domain integral swapping," Microwave and Optical Technology Letters, Vob. 49, No. 3, pp. 709-712, March 2007

[22] J. M. Rius, E. Ubeda, and J. Parrón, "On the testing of the magnetic field integral equation with rwg basis functions in method of moments," IEEE Transactions on Antennas and Propagation,, vol. 49, no. 11, pp. 1550-1553, November 2001.

[23] Özgur Ergül, and Levent Gürel, "Linear-Linear Basis Functions for MLFMA Solutions of Magnetic-Field and Combined-Field Integral Equations," IEEE Transactions on Antennas and Propagation, Vol. 55, No. 4, pp. 1103-1110, April 2007

[24] Jonas Kornprobst and Thomas F. Eibert, "An Accurate Low-Order Discretization Scheme for the Identity Operator in the Magnetic Field and Combined Field Integral Equations,” IEEE Trans. Antennas Propag., Vol. 66, No. 11, pp. 6146- 6157, November 2018.

[25] E. Ubeda and J. M. Rius, "MFIE MoM-formulation with curl-conforming basis functions and accurate Kernel-integration in the analysis of perfectly conducting sharp-edged objects", Microwave and Optical Technology letters, vol. 44, No. 4, February 2005.

[26] Ö. Ergül and L. Gürel, "The use of curl-conforming basis functions for the magnetic-field integral equation," IEEE Transactions on Antennas and Propagation, vol. 54, no. 7, pp. 1917-1926, Jul. 2006.

[27] E. Ubeda, José M. Tamayo and Juan M. Rius, "Taylor-Orthogonal basis functions for the discretizations in method of moments of second kind integral equations in the scattering analysis of perfectly conducting or dielectric objects", Progress in Electromagnetics Research, vol. 119, 85-105, 2011.

[28] K. Cools, F. P. Andriulli, D. De Zutter, and E. Michielssen, "Accurate and Conforming Mixed Discretization of the MFIE," IEEE Antennas and wireless propagation letters, vol. 10, 2011.

[29] Liming Zhang, Ali Deng, Yiqing Zhang, Xianzhu Meng and Zengtao Lv, "Electromagnetic scattering analysis using nonconformal meshes and monopolar curl-conforming basis functions," Engineering Analysis with Boundary Elements, vol. 65, pp. 47-54, 2016 
[30] Eduard Ubeda,, Jose M. Tamayo and Juan M. Rius, "Orthogonal basis functions for the discretization of the magnetic-field integral equation in the low frequency regime ," European Conference on Antennas and Propagation, 12-16 April 2010, Barcelona (Spain).

[31] Pasi Ylä-Oijala and Matti Taskinen," "Calculation of CFIE impedance matrix elements with RWG and nxRWG functions," IEEE Transactions on Antennas and Propagation, vol. 51, no. 8, pp. 1837-1846, August 2003.

[32] Su Yan, Jian-Ming Jin, and Zaiping Nie, "Improving the accuracy of the second-kind fredholm integral equations by using the BuffaChristiansen functions," IEEE Transactions on Antennas and Propagation, vol. 59, no. 4, pp. 1299-1310, April 2011.

[33] C. A. Balanis, Advanced Engineering Electromagnetics. New York: Wiley, 1989.

[34] D. H. Schaubert, D. R. Wilton and A. W. Glisson, "A Tetrahedral Modeling for Electromagnetic Scattering by arbitrarily shaped Inhomogeneous Dielectric Bodies”, IEEE Transactions on Antennas and Propagation, vol. AP-32, No. 1, January 1984.

[35] Eduard Ubeda, Juan M. Rius and Alex Heldring, "Nonconforming discretization of the Magnetic-Field Integral Equation with volumetric testing," IEEE Antennas and Propagation Society International Symposium, 6-11 July 2014, Memphis (TN)

[36] Ivan Sekulic, Eduard Ubeda and Juan M. Rius, "Improved accuracy in the scattering analysis of arbitrarily shaped ferromagnetic objects," 2017 IEEE MTT-S International Conference on Numerical Electromagnetic and Multiphysics Modeling and Optimization for RF, Microwave, and Terahertz Applications (NEMO), 17-19 May 2017, Sevilla (Spain)

[37] Zhen Peng, Xiao-Chuan Wang, and Jin-Fa Lee, "Integral equation based domain decomposition method for solving electromagnetic wave scattering from non-penetrable objects, ” IEEE Transactions on Antennas and Propagation, vol.. 59, No. 9, pp. 3328-3338, September 2011

[38] Z. Peng, R. Hiptmair,Y. Shao, and B.MacKie-Mason, "Domain decomposition preconditioning for surface integral equations in solving challenging electromagnetic scattering problems," IEEE Transactions on Antennas and Propagation, vol. 64, no. 1, pp. 210-223, Jan. 2016.

[39] Kui Han, Yongpin Chen, Xiaofeng Que, Ming Jiang Jun Hu and Zaiping Nie, "A Domain Decomposition Scheme With Curvilinear Discretizations for Solving Large and Complex PEC Scattering Problems", IEEE Transactions on Antennas and Propagation", Vol. 17, No. 2, pp. $242-246$, february 2018

[40] D. R. Wilton, S. M. Rao, A. W. Glisson, D. H. Schaubert, O. M. Al-Bundak, and C. M. Butler, "Potential integrals for uniform and linear source distributions on polygonal and polyhedral domains," IEEE Transactions on Antennas and Propagation, vol. AP-32, no. 3, pp. 276281, March 1984.

[41] D. A. Dunavant, "High degree efficient symmetrical Gaussian quadrature rules for the triangle," Int. J. Numer. Meth. Eng., vol. 21, pp. $1129-1148,1985$.

[42] M. Gellert and R. Harbord, "Moderate degree cubature formulas for 3-D tetrahedral finite element approximations," Commun. Appl. Numer. Meth., vol. 7, pp. 487-495, 1991.

[43] Z. G. Qian and W. C. Chew, “A quantitative study on the low frequency breakdown of EFIE,” vol. 50, no. 5, pp. 1159-1162.

[44] Z.-G. Qian and W. C. Chew, "Enhanced A-EFIE With Perturbation Method,” vol. 58, no. 10, pp. 3256-3264.

[45] Yunhua Zhang, Tie Jun Cui, Weng Cho Chew, and Jun-Sheng Zhao, "Magnetic field integral equation at very low frequencies," vol. 51, no. 8, pp. 1864-1871.

[46] E. Ubeda and J. M. Rius, "Divergence-conforming discretization of second-kind integral equations for the RCS computation in the Rayleigh frequency region," Radio Sci., vol. 46, no. 5, p. RS5005, 2011, doi: 10.1029/2010RS004550.

[47] G. H. Golub and C. F. Van Loan, Matrix computations, The Johns Hopkins University Press, 1996.

[48] J. Jin, The Finite Element Method in Electromagnetics. John Wiley and Sons, 1993.

[49] A. F. Peterson, "Observed baseline convergence rates and superconvergence in the scattering cross Section obtained from numerical solutions of the MFIE potential integrals for uniform and linear source distributions on polygonal and polyhedral domains," IEEE Transactions on Antennas and Propagation, vol. 56, no. 11, pp. 3510-3515, November 2008.

[50] K. Cools, F. P. Andriulli, F. Olyslager and E. Michielssen, "Nullspaces of MFIE and Calderón Preconditioned EFIE Operators Applied to Toroidal Surfaces," IEEE Transactions on Antennas and Propagation, vol. 57, no. 10, pp. 3205-3215, October 2009.

[51] W. E. Blore, "The Radar Cross Section of Ogives, Double-Backed Cones, Double-Kounded Cones, and Cone Spheres," IEEE Transactions on Antennas and Propagation, vol AP-12, Sep 1964, pp 582-590. 